\title{
SPECTRAL CONVERGENCE OF NON-COMPACT QUASI-ONE-DIMENSIONAL SPACES
}

\author{
OLAF POST
}

\begin{abstract}
We consider a family of non-compact manifolds $X_{\varepsilon}$ ("graph-like manifolds") approaching a metric graph $X_{0}$ and establish convergence results of the related natural operators, namely the (Neumann) Laplacian $\Delta_{X_{\varepsilon}}$ and the generalised Neumann (Kirchhoff) Laplacian $\Delta_{X_{0}}$ on the metric graph. In particular, we show the norm convergence of the resolvents, spectral projections and eigenfunctions. As a consequence, the essential and the discrete spectrum converge as well. Neither the manifolds nor the metric graph need to be compact, we only need some natural uniformity assumptions. We provide examples of manifolds having spectral gaps in the essential spectrum, discrete eigenvalues in the gaps or even manifolds approaching a fractal spectrum. The convergence results will be given in a completely abstract setting dealing with operators acting in different spaces, applicable also in other geometric situations.
\end{abstract}

\section{INTRODUCTION}

The aim of this article is to show that non-compact quasi-one-dimensional spaces can be approximated by the underlying metric graph. A metric or quantum graph is a graph considered as one-dimensional space where each edge is assigned a length. A quasi-one-dimensional space consists of a family of graphlike manifolds, i.e., a family of manifolds $X_{\varepsilon}$ shrinking to the underlying metric graph $X_{0}$. The family of graph-like manifolds is constructed of building blocks $U_{\varepsilon, v}$ and $U_{\varepsilon, e}$ for each vertex $v \in V$ and edge $e \in E$ of the graph, respectively (cf. Figure (1). The cross section of the edge neighbourhood $U_{\varepsilon, e}$ as well as the boundary component of $U_{\varepsilon, v}$, where $U_{\varepsilon, e}$ meet, consists of a manifold $F_{\varepsilon}$ with radius of order $\varepsilon$. The cross section could have a boundary resulting in a manifold $X_{\varepsilon}$ with boundary. In addition, the vertex neighbourhoods $U_{\varepsilon, v}$ are assumed to be small. The simplest example is the $\varepsilon$-neighbourhood $X_{\varepsilon}$ of a quantum graph $X_{0}$ embedded in $\mathbb{R}^{2}$. In this case, the cross section is $F_{\varepsilon}=(-\varepsilon, \varepsilon)$. A simple boundaryless example is given by the surface of a pipeline network according to the underlying graph $X_{0}$ (cf. Figure 21). Here, the cross section consists of a circle of radius $\varepsilon$.

On the graph-like manifold $X_{\varepsilon}$ we consider the Laplacian $\widetilde{H}:=\Delta_{X_{\varepsilon}} \geq 0$ acting in the Hilbert space $\widetilde{\mathcal{H}}:=\mathrm{L}_{2}\left(X_{\varepsilon}\right)$. If $X_{\varepsilon}$ has a boundary, we impose Neumann boundary conditions. On the graph, we choose the natural Laplacian $H:=\Delta_{X_{0}} \geq 0$, namely, the generalised Neumann (Kirchhoff) Laplacian acting on each edge as a one-dimensional weighted Laplacian (cf. Eq. (2.2)). On each vertex, we assume continuity and current conservation (cf. Eq. (2.3)). Note that $\Delta_{X_{0}}$ acts on $\mathcal{H}:=\oplus_{e} \mathrm{~L}_{2}(e)$ where each edge $e$ is identified with the interval 

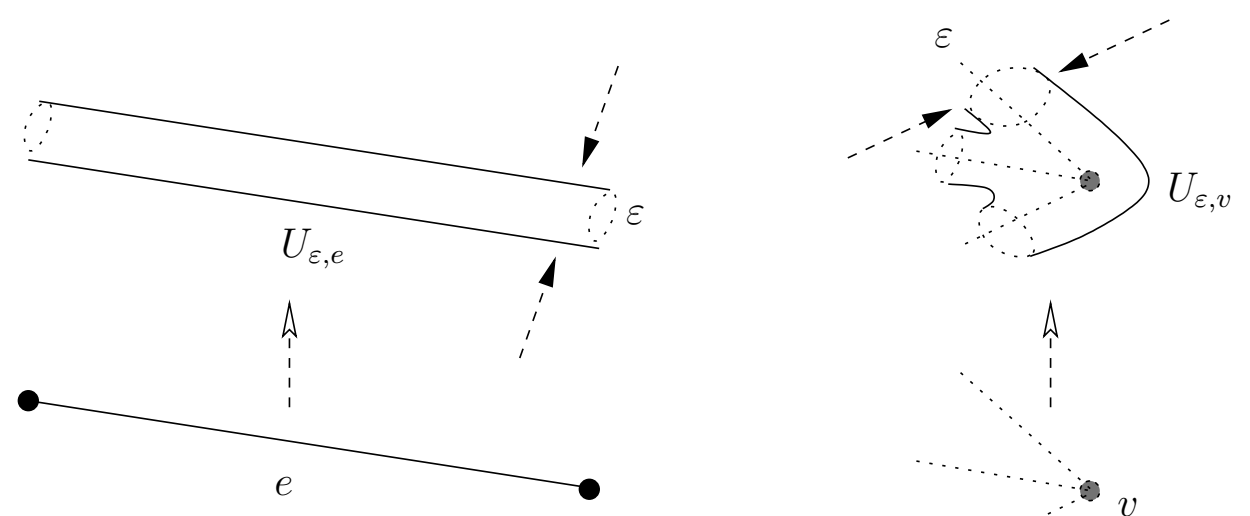

FiguRE 1. The associated edge and vertex neighbourhoods with $F_{\varepsilon}=\mathbb{S}_{\varepsilon}^{1}$, i.e., $U_{\varepsilon, e}$ and $U_{\varepsilon, v}$ are 2-dimensional manifolds with boundary.
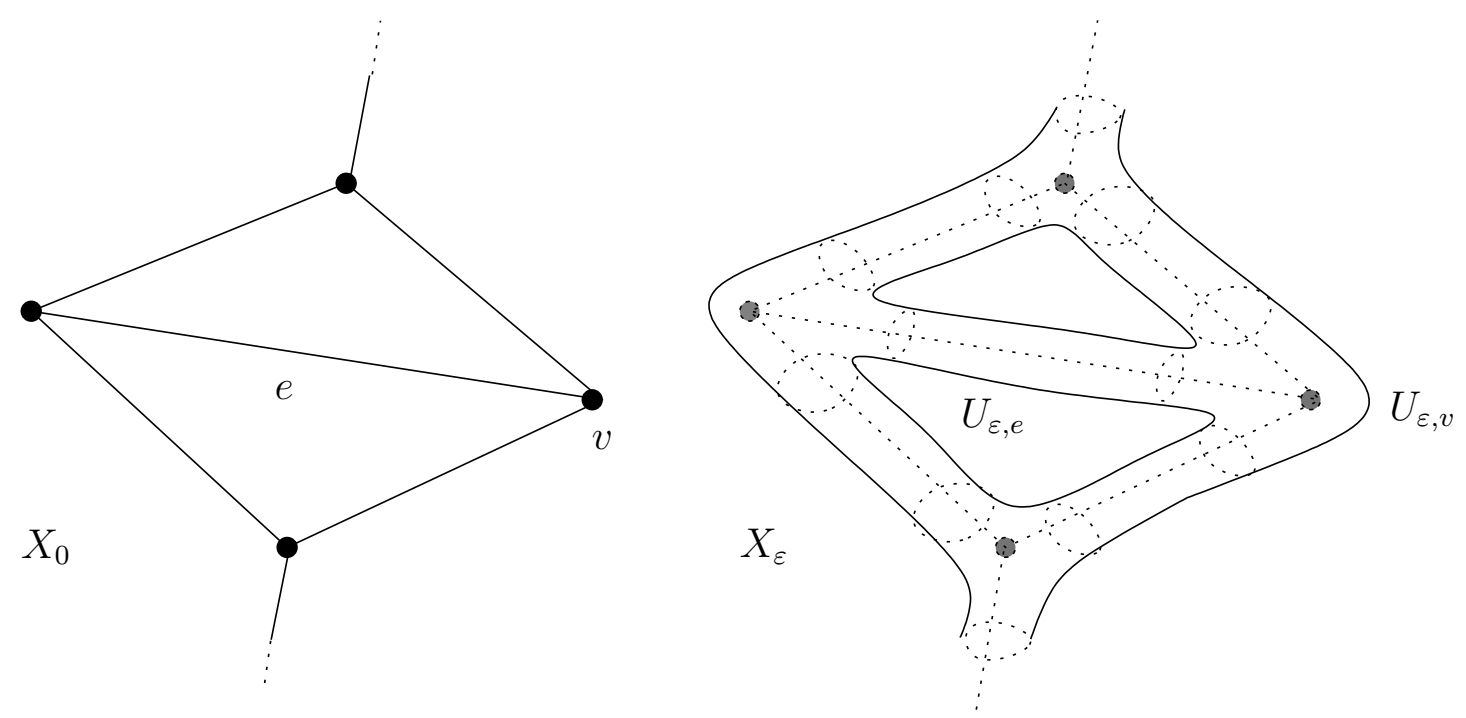

Figure 2. On the left, we have the graph $X_{0}$, on the right, the associated family of graph-like manifolds. Here, $F_{\varepsilon}=\mathbb{S}_{\varepsilon}^{1}$ is the transversal section of radius $\varepsilon$ and $X_{\varepsilon}$ is a 2-dimensional manifold.

$\left(0, \ell_{e}\right)\left(0<\ell_{e} \leq \infty\right)$ - in contrast to the discrete graph Laplacian acting as difference operator on the space of vertices, $\ell_{2}(V)$. For a relation between these two operators see Section 3.3.

In this article, we concentrate on the spectrum of such systems. Our main result is the following:

Main Theorem (Theorem 2.13). Suppose $X_{\varepsilon}$ is a family of (non-compact) graph-like manifolds associated to a metric graph $X_{0}$. If $X_{\varepsilon}$ and $X_{0}$ satisfy some natural uniformity conditions, then the resolvent of $\Delta_{X_{\varepsilon}}$ converges in norm to the resolvent of $\Delta_{X_{0}}$ (with suitable identification operators) as $\varepsilon \rightarrow 0$. In particular, the corresponding essential and discrete spectra converge uniformly in any bounded interval. Furthermore, the eigenfunctions converge as well. 
The uniformity conditions are precisely stated in Section 2.4. For example we need a global lower bound on the edge length $\ell_{e} \geq \ell_{0}$ and a global upper bound on the vertex degree $\operatorname{deg} v \leq d_{0}$. In the case when the graph $X_{0}$ is embedded in $\mathbb{R}^{2}$ (cf. Section 3.1) the uniformity conditions mean in particular, that we need a global bound on the curvature of an edge and a global lower bound on the angle between two different edges at a vertex although both quantities do not enter into the limit operator and space.

In contrast to previous articles (cf. [RS01, KuZ01, KuZ03, EP05]) we allow here infinite structures, i.e., we drop the condition of compactness of $X_{\varepsilon}$ and $X_{0}$. Therefore, we cannot use the variational principle in order to characterise the discrete spectrum. The appropriate substitute is an abstract convergence criterion provided in Appendix A. The basic idea is to define a "distance" between the operators $\Delta_{X_{0}}$ and $\Delta_{X_{\varepsilon}}$ with suitable identification operators (cf. Definition A.1). We have formulated the abstract results fully in terms of this "distance" in order to trace the parameter dependence on $\varepsilon$ of the operator $\Delta_{X_{\varepsilon}}$, the Hilbert space $\mathrm{L}_{2}\left(X_{\varepsilon}\right)$ and the identification operators between the graph and the manifold. The "distance" can be calculated in terms of the associated sesquilinear forms which makes the verification quite simple in our main model. As a consequence, we show norm resolvent convergence which implies all other convergence results like convergence of the spectral projections, convergence of the eigenfunctions and convergence of the spectra. Note that in RS01, KuZ01, KuZ03, EP05, only convergence of eigenvalues has been established. Our results here show, that the eigenvectors converge as well. We will show in a forthcoming paper that this abstract convergence criterium has applications in other geometrical situations.

A related result on non-compact spaces has been established in Sa00. Saito considered metric trees (allowing also arbitrary small edges, i.e., no lower bound on $\ell_{e}$ ) together with a suitable $\varepsilon$-neighbourhood, but showed only weak convergence of the resolvents. In [EvS00] the authors prove exact relations (equality, inclusion) of the essential spectrum of the Neumann Laplacian on a thickened tree (for fixed $\varepsilon$ in our notation) and the corresponding metric graph.

Our spectral convergence result has many applications in different situations: First, we can consider graph-like manifolds as a kind of toolbox in order to construct manifolds with prescribed spectrum, at least approximately. For example, we are able to construct manifolds with gaps in the essential spectrum (cf. Thms. 3.4, 3.5 ) also in the non-periodic case: Using the recent result on graph decoration one can construct metric graphs with spectral gaps (cf. [AS00, BEG03, BGL05, Ku05, and Section 3.2). Our spectral convergence result then immediately states that an associated graph-like manifold also has gaps. In the periodic case (i.e., on covering spaces with compact quotient), we have of course the same result once we ensure the existence of gaps on the quantum graph. For the existence of spectral gaps on periodic manifolds (not necessarily graph-like in our sense) we refer to [P03b, LP04, LP05] and the references therein. The periodic case can often be reduced to the spectral convergence on a compact space.

An example with arbitrary many gaps in a compact spectral interval is given by a fractal-like manifold in Theorem 3.10. The graph-like manifold is constructed according to a Sierpiński graph. It was shown in [T98 that the discrete Laplacian 
on a Sierpiński graph has pure point spectrum which is purely essential and of fractal nature. Using a nice relation between the spectrum of the discrete graph Laplacian and the metric graph Laplacian with constant edge length $\ell_{e}=\ell$ developed in [Ca97] (cf. Theorem 3.6) we are able to construct a family of graphlike manifolds $X_{\varepsilon}$ such that the spectrum of $\Delta_{X_{\varepsilon}}$ approaches a fractal set. In particular, the spectrum of $\Delta_{X_{\varepsilon}}$ has an arbitrary (a priori finite) number of spectral gaps in the compact interval $[0, \Lambda]$ provided $\varepsilon$ is small enough. Such fractal manifolds have been constructed in [BCG01] in order to provide examples of smooth spaces sharing properties of fractal spaces in large scales (e.g. heat kernel estimates).

Finally, our result shows rigorously, that the physically intuition of modeling quasi-one-dimensional spaces by its singular limit is correct, also on infinite structures. Graph models have a long history in modeling properties of networks, complicated organic molecules RuS53 or quite recently, nanostructures, i.e., structures, which are too small to be considered classically, but still too large to be described on a conventional quantum level, see e.g. AGHH05, KoS99, Ku02, $\mathrm{Ku} 04, \mathrm{Ku} 05$. On the one side quantum graphs provide a solvable model in quantum mechanics in the sense that many quantities can be calculated explicitely essentially by solving systems of ODEs. On the other side, the structure of a metric graph is still rich enough in order to provide a good model for branched structures. For example, a spectral gap corresponds to "forbidden modes", i.e., a particle with an energy in the gap cannot propagate through the system. In this sense, transport properties on $X_{\varepsilon}$ are approximately described by the quantum graph $X_{0}$. Furthermore, a bound state (of finite degeneracy) on $X_{\varepsilon}$ (i.e., an eigenfunction corresponding to a discrete eigenvalue) can be approximated by its (mostly explicitly known) eigenvector on $X_{0}$ (cf. Theorem 3.5). In a forthcoming paper [EP06] we will deal with the convergence of resonances, i.e., eigenvalues of a suitable dilated Hamiltonian. The methods needed there differ from the ones given in Appendix $\mathrm{A}$ since the dilated operators are no longer self-adjoint (even not normal).

With our methods here, we consider the discrete and the essential spectrum only since they can be characterised by the dimension of spectral projections. A finer analysis of the spectrum needs more elaborated methods, such as scattering theory. Our results presented here are considered as a first step in dealing with the above-mentioned structures. We will concentrate on the relation between scattering and transport properties on the two systems in a forthcoming paper.

The paper is organised as follows. In Section 2 we define properly graph-like manifolds and metric graphs and show that the abstract convergence result can be used in this situation for $(H, \mathcal{H})=\left(\Delta_{X_{0}}, \mathrm{~L}_{2}\left(X_{0}\right)\right)$ and $(\widetilde{H}, \widetilde{\mathcal{H}})=\left(\Delta_{X_{\varepsilon}}, \mathrm{L}_{2}\left(X_{\varepsilon}\right)\right)$. In Section 3 we discuss various examples of graph-like manifolds to which our result applies. We also derive several consequences of the spectral convergence. In Appendix $\mathrm{A}$ we develop the abstract framework in order to show the spectral convergence for arbitrary pairs $(H, \mathcal{H})$ and $(\widetilde{H}, \widetilde{\mathcal{H}})$ being at a "distance" $\delta$ to each other. 


\section{GRAPH-LIKE MANIFOLDS}

In this section we apply the abstract setting developed in Appendix A to the example of a family of manifolds $X_{\varepsilon}$ converging to a (metric) graph $X_{0}$. This situation has already been treated in a quite general way in [EP05] based on KuZ01, RS01 with the only restriction that the graph is compact (i.e., finite and each edge has finite length) and each manifold $X_{\varepsilon}$ is compact. Under these assumptions, the spectra of the operators considered are purely discrete (for a precise definition see below). The main result in EP05] states that the $k$-th eigenvalue of the Laplacian $\Delta_{X_{\varepsilon}}$ converges to the $k$-th eigenvalue of the limit operator. The proof uses the min-max principle and comparison of the appropriate Rayleigh quotients.

If the manifold and the metric graph are non-compact, more elaborated methods are needed. Namely, we establish in Appendix A norm resolvent convergence from which all other convergence results follow. The norm resolvent convergence is reduced to the verification of several natural conditions provided in Definition A.1. In order that these conditions are satisfied we need the uniformity assumptions (G1) -G7) in our model. The eigenvalue convergence already proven in [EP05] appears as a special case (cf. Corollary A.15).

2.1. Metric graphs. Let us first describe the metric graph $X_{0}$ and the family of graph-like manifolds $X_{\varepsilon}$; the necessary assumptions in order that the convergence results hold will be given later. Suppose $X_{0}=(V, E, \partial, \ell)$ is a countable, connected metric graph, i.e., $V$ denotes the set of vertices, $E$ the set of edges and $\partial: E \longrightarrow V \times V, \partial e=\left(\partial_{+} e, \partial_{-} e\right)$ denotes the pair of the end point and the starting point of the edge $e$. For each vertex $v \in V$ we denote by

$$
E_{v}^{ \pm}:=\left\{e \in E \mid \partial_{ \pm} e=v\right\}
$$

the edges starting $(-) /$ ending $(+)$ at $v$. Let $E_{v}:=E_{v}^{+} \uplus E_{v}^{-}$be the disjoint union of all edges emanating at $v$. The degree of a vertex $v$ is the number of vertices emanating from $v$, i.e.,

$$
\operatorname{deg} v:=\left|E_{v}\right|=\left|E_{v}^{+}\right|+\left|E_{v}^{-}\right| .
$$

We assume that $X_{0}$ is locally finite, i.e., $\operatorname{deg} v \in \mathbb{N}$. Note that we allow loops, i.e. edges $e$ with $\partial_{+} e=\partial_{-} e=v$. A loop $e$ will be counted twice in $\operatorname{deg} v$ and occurs twice in $E_{v}$ due to the disjoint union. In addition, we assume that $\partial e$ always consists of two elements, even if $\partial_{-} e=\partial_{+} e=v$ for a loop $e$. We also allow multiple edges, i.e., edges $e_{1} \neq e_{2}$ having the same starting and end points.

Finally, $\ell: E \longrightarrow(0, \infty]$ assigns a length $\ell_{e}$ to each edge $e \in E$, making the graph $(V, E, \partial)$ a metric or quantum graph. Clearly, $X_{0}$ becomes a metric space. We identify each edge $e$ with the interval $\left(0, \ell_{e}\right)$. In the case of an infinite edge, a "lead", (i.e., $\ell_{e}=\infty$ ) we assume that there is only one vertex $\partial e=\partial_{-} e$ at the end corresponding to 0 , i.e., there is no vertex at $\infty$. For a general survey on quantum graphs consult e.g. Ku04, Ku05. We stress that our graphs need by no way to be embedded in some Euclidean space.

Remark 2.1. Note that for a metric graph, the notion "compact" and "finite" have a different meaning: A finite metric graph is a graph with finitely many vertices and edges, whereas a compact graph must in addition have finite edge length for 
each edge. Therefore, a compact metric graph is finite but not vice versa (think e.g. of a star-shaped metric graph with one vertex and a finite number of leads attached to the vertex).

We also assign a density $p_{e}$ to each edge $e \in E$, i.e., a measurable function $p_{e}: e \longrightarrow(0, \infty)$. For simplicity, we assume that $p_{e}$ is smooth in order to obtain a smooth metric in the graph-like manifold. The data $(V, E, \partial, \ell, p), p=\left(p_{e}\right)_{e}$ describe a weighted metric graph.

The Hilbert space associated to such a graph is

$$
\mathcal{H}:=\mathrm{L}_{2}\left(X_{0}\right)=\bigoplus_{e \in E} \mathrm{~L}_{2}(e)
$$

which consists of all functions $f$ with finite norm

$$
\|f\|^{2}=\|f\|_{X_{0}}^{2}=\sum_{e \in E}\left\|f_{e}\right\|_{e}^{2}=\sum_{e \in E} \int_{e}\left|f_{e}(x)\right|^{2} p_{e}(x) \mathrm{d} x .
$$

We define the limit operator $H$ via the quadratic form

$$
\mathfrak{h}(f):=\sum_{e \in E}\left\|f_{e}^{\prime}\right\|_{e}^{2}=\sum_{e \in E} \int_{e}\left|f_{e}^{\prime}(x)\right|^{2} p_{e}(x) \mathrm{d} x
$$

for functions $f$ in

$$
\mathcal{H}_{1}:=\mathrm{H}^{1}\left(X_{0}\right):=\mathrm{C}\left(X_{0}\right) \cap \bigoplus_{e \in E} \mathrm{H}^{1}(e) .
$$

Note that a weakly differentiable function on an interval $e$, i.e., $f_{e} \in \mathrm{H}^{1}(e)$, is automatically continuous. Therefore, the continuity is only a condition at each vertex. Furthermore, $\mathfrak{h}$ is a closed form, i.e., $\mathcal{H}_{1}$ together with the norm

$$
\|f\|_{1}^{2}=\|f\|_{1, X_{0}}^{2}:=\|f\|_{X_{0}}^{2}+\mathfrak{h}(f)
$$

is complete.

The associated self-adjoint, non-negative operator $H=\Delta_{X_{0}}$ is given by

$$
\left(\Delta_{X_{0}} f\right)_{e}=-\frac{1}{p_{e}}\left(p_{e} f_{e}^{\prime}\right)^{\prime}
$$

on each edge $e$. If we assume the global lower bound (G2) (cf. page 9) on the length $\ell_{e}$ of the edge $e$ then the domain $\mathcal{H}_{2}$ of $H=\Delta_{X_{0}}$ consists of all functions $f \in \mathrm{L}_{2}\left(X_{0}\right)$ such that $\Delta_{X_{0}} f \in \mathrm{L}_{2}\left(X_{0}\right)$ (cf. e.g. [Ku04, Thm. 17]). Furthermore, each function $f$ satisfies the so-called (generalised) Neumann boundary condition (sometimes also named Kirchhoff) at each vertex $v$, i.e., $f$ is continuous at $v$ and

$$
\sum_{e \in E_{v}} p_{e}(v) f_{e}^{\prime}(v)=0
$$

for all vertices $v \in V$ where the derivative is taken away from the vertex, i.e. we set $f_{e}^{\prime}(v):=f_{e}^{\prime}(0)$ if $v=\partial_{-} e$ and $f_{e}^{\prime}(v):=-f_{e}^{\prime}\left(\ell_{e}\right)$ if $v=\partial_{+} e$ (considering $f_{e}$ as function on the interval $\left.\left(0, \ell_{e}\right)\right)$. We will call $\Delta_{X_{0}}$ the (generalised) weighted Neumann Laplacian on $X_{0}$. For details on operators on non-compact or infinite metric graphs we refer e.g. to $\mathrm{Ku} 04, \mathrm{Ku} 05$. 
2.2. Graph-like manifolds. Let $X_{0}$ be a weighted metric graph as defined in the previous section. The corresponding family of graph-like manifolds $X_{\varepsilon}$ is given as follows: For each $0<\varepsilon \leq \varepsilon_{0}$ we associate with the graph $X_{0}$ a connected Riemannian manifold $X_{\varepsilon}$ of dimension $d \geq 2$ with or without boundary equipped with a metric $g_{\varepsilon}$ to be specified below ${ }^{1}$. We suppose that $X_{\varepsilon}$ is the union of the closure of open subsets $U_{\varepsilon, e}$ and $U_{\varepsilon, v}$ such that the $U_{\varepsilon, e}$ and $U_{\varepsilon, v}$ are mutually disjoint for all possible combinations of $e \in E$ and $v \in V$, i.e.,

$$
X_{\varepsilon}=\bigcup_{e \in E} \bar{U}_{\varepsilon, e} \cup \bigcup_{v \in V} \bar{U}_{\varepsilon, v}
$$

We think of $U_{\varepsilon, e}$ as the thickened edge $e$ and of $U_{\varepsilon, v}$ as the thickened vertex $v$ (see Figures 1 and 21). Note that Figure 2 describes the situation only roughly, since it assumes that $X_{\varepsilon}$ is embedded in $\mathbb{R}^{\nu}$. More correctly, we should think of $X_{\varepsilon}$ as an abstract manifold obtained by identifying the appropriate boundary parts of $U_{\varepsilon, e}$ and $U_{\varepsilon, v}$ via the connection rules of the graph $X_{0}$. This manifold need not to be embedded, but the situation when $X_{\varepsilon}$ is a submanifold of $\mathbb{R}^{\nu}(\nu \geq d)$ can be viewed also in this abstract context; note that the $\varepsilon$-neighbourhood of an embedded metric graph in $\mathbb{R}^{d}$ is also included as example (cf. EP05] and Section 3.1).

As a matter of convenience we assume that $U_{\varepsilon, e}$ and $U_{\varepsilon, v}$ are independent of $\varepsilon$ as manifolds, i.e., only their metrics $g_{\varepsilon}$ depend on $\varepsilon$. This can be achieved in the following way: for the edge regions we assume that $U_{\varepsilon, e}$ is diffeomorphic to $U_{e}:=e \times F$ for all $0<\varepsilon \leq \varepsilon_{0}$ where $F$ denotes a compact and connected manifold (with or without a boundary) of dimension $m:=d-1$. We fix a metric $h$ on $F$ and assume for simplicity that vol $F=1$.

For the vertex regions we assume that the manifold $U_{\varepsilon, v}$ is diffeomorphic to an $\varepsilon$-independent manifold $U_{v}$ for $0<\varepsilon \leq \varepsilon_{0}$. Pulling back the metric to the diffeomorphic manifold $U_{e}$ resp. $U_{v}$ we may assume that the underlying differentiable manifold is independent of $\varepsilon$. Therefore, $U_{\varepsilon, e} \cong\left(U_{e}, g_{\varepsilon, e}\right)$ and $U_{\varepsilon, v}=\left(U_{v}, g_{\varepsilon, v}\right)$.

We use the obvious notation for functions $u$ on $X_{\varepsilon}$ like $u_{e}$ and $u_{v}$ as restrictions on $U_{\varepsilon, e}$ and $U_{\varepsilon, v}$, respectively. The corresponding Hilbert space is then

$$
\widetilde{\mathcal{H}}:=\mathrm{L}_{2}\left(X_{\varepsilon}\right)=\bigoplus_{e \in E} \mathrm{~L}_{2}\left(U_{\varepsilon, e}\right) \oplus \bigoplus_{v \in V} \mathrm{~L}_{2}\left(U_{\varepsilon, v}\right)
$$

which consists of all functions $u$ with finite norm

$$
\begin{aligned}
\|u\|^{2}=\|u\|_{X_{\varepsilon}}^{2}=\sum_{e \in E} \| & u_{e}\left\|_{U_{\varepsilon, e}}^{2}+\sum_{v \in V}\right\| u_{v} \|_{U_{\varepsilon, v}}^{2} \\
& =\sum_{e \in E} \int_{e \times F}\left|u_{e}\right|^{2} \operatorname{det} g_{\varepsilon, e}^{1 / 2} \mathrm{~d} x \mathrm{~d} y+\sum_{v \in V} \int_{U_{v}}\left|u_{v}\right|^{2} \operatorname{det} g_{\varepsilon, v}^{1 / 2} \mathrm{~d} z
\end{aligned}
$$

where $y$ and $z$ represent coordinates of $F$ and $U_{v}$, respectively.

The operator $\widetilde{H}$ we are considering will be the Laplacian on $X_{\varepsilon}$, i.e., $\widetilde{H}=\Delta_{X_{\varepsilon}}$. If $F$ has non-trivial boundary, we assume Neumann boundary conditions on the

\footnotetext{
${ }^{1}$ The boundary of $X_{\varepsilon}$ (if there is any) need not to be smooth; we allow singularities on the boundary of the vertex neighbourhood $U_{\varepsilon, v}$, see e.g. Section 3.1 and Figure 3
} 
boundary part coming from $\partial F$. We define $\Delta_{X_{\varepsilon}}$ via its quadratic form $\widetilde{\mathfrak{h}}$ given by

$$
\widetilde{\mathfrak{h}}(u)=\int_{X_{\varepsilon}}|\mathrm{d} u|_{g_{\varepsilon}}^{2} \mathrm{~d} X_{\varepsilon}
$$

for functions $u \in \widetilde{\mathcal{H}}_{1}=\mathrm{H}^{1}\left(X_{\varepsilon}\right)$ where the latter space denotes the completion of the space of smooth functions with bounded support w.r.t. the norm

$$
\|u\|_{1}^{2}=\|u\|_{1, X_{\varepsilon}}^{2}:=\|u\|_{X_{\varepsilon}}^{2}+\widetilde{\mathfrak{h}}(u) .
$$

2.3. Quasi-unitary operators. Let us fix the identification operators $J: \mathcal{H} \longrightarrow$ $\widetilde{\mathcal{H}}$ and $J^{\prime}: \widetilde{\mathcal{H}} \longrightarrow \mathcal{H}$ and their analogues on the quadratic form domains. Roughly speaking, $J$ extends the function $f$ at $x \in e$ constantly onto the cross section $F_{\varepsilon, e}(x):=\left(\{x\} \times F, h_{\varepsilon, e}\right) \subset\left(U_{e}, g_{\varepsilon, e}\right)$, where $h_{\varepsilon, e}$ is the induced metric of the restriction, and $J^{\prime}$ is the transversal average of $u$ at $x$, i.e., the Fourier coefficient of $u(x, \cdot)$ w.r.t. the first (constant) eigenfunction on $F_{\varepsilon, e}(x)$. We will first show what estimates are necessary in order that $J, J^{\prime}$ and their quadratic form analogues become quasi-unitary in the sense of Definition A.1. In a second step we provide the necessary assumptions on the graph (Section 2.4) and on the manifold (Section [2.5). Finally, we provide some necessary estimates (Section 2.6) and finish the proof of quasi-unitarity.

We define the operator $J: \mathcal{H} \longrightarrow \widetilde{\mathcal{H}}$ by

$$
J f(z):= \begin{cases}\varepsilon^{-m / 2} f_{e}(x) & \text { if } z=(x, y) \in U_{e}, \\ 0 & \text { if } z \in U_{v}\end{cases}
$$

and the operator $J_{1}: \mathcal{H}_{1} \longrightarrow \widetilde{\mathcal{H}}_{1}$ by

$$
J_{1} f(z):= \begin{cases}\varepsilon^{-m / 2} f_{e}(x) & \text { if } z=(x, y) \in U_{e}, \\ \varepsilon^{-m / 2} f(v) & \text { if } z \in U_{v}\end{cases}
$$

Note that the latter operator is well-defined since functions in $\mathcal{H}_{1}$ are continuous (cf. Lemma 2.4). For the operators in the opposite direction, we first introduce the following averaging operators

$$
\begin{aligned}
\left(N_{e} u\right)(x) & :=\left\langle\varphi_{F, 1}, u_{e}(x, \cdot)\right\rangle_{F}=\int_{F} u_{e}(x, y) \mathrm{d} F(y), \\
C_{v} u & :=\left\langle\varphi_{U_{v}, 1}, u_{v}\right\rangle_{U_{v}}=\frac{1}{\operatorname{vol} U_{v}} \int_{U_{v}} u \mathrm{~d} U_{v}
\end{aligned}
$$

for $u \in \widetilde{\mathcal{H}}=\mathrm{L}_{2}\left(X_{\varepsilon}\right)$ giving the coefficient corresponding to the first (transversal) eigenfunction $\varphi_{1}$ on $U_{e}$ resp. $U_{v}$. Note that these eigenfunctions are constant and that vol $F=1$.

We define $J^{\prime}: \widetilde{\mathcal{H}} \longrightarrow \mathcal{H}$ by

$$
\left(J^{\prime} u\right)_{e}(x):=\varepsilon^{m / 2}\left(N_{e} u\right)(x), \quad x \in e
$$

and the operator $J_{1}^{\prime}: \widetilde{\mathcal{H}}_{1} \longrightarrow \mathcal{H}_{1}$ by

$$
\begin{aligned}
\left(J_{1}^{\prime} u\right)_{e}(x):= & \varepsilon^{m / 2}\left[N_{e} u(x)\right. \\
& \left.+\rho_{e}^{+}(x)\left[C_{\partial_{+} e} u-N_{e} u\left(\partial_{+} e\right)\right]+\rho_{e}^{-}(x)\left[C_{\partial_{-} e} u-N_{e} u\left(\partial_{-} e\right)\right]\right]
\end{aligned}
$$


for $x \in e$. Here, $\rho_{e}^{ \pm}: \mathbb{R} \longrightarrow[0,1]$ are the continuous, piecewise affine functions given by

$$
\rho_{e}^{+}\left(\partial_{+} e\right)=1 \quad \text { and } \quad \rho_{e}^{+}(x)=0 \quad \text { for all } \operatorname{dist}\left(x, \partial_{+} e\right) \geq \min \left\{1, \ell_{e} / 2\right\}
$$

and similarly for $\rho_{e}^{-}$and $\partial_{-} e$. Note that $\left(J_{1}^{\prime} u\right)_{e}(v)=C_{v} u$ for $v=\partial_{ \pm} e$. In particular, $J_{1}^{\prime} u$ is a continuous function on $X_{0}$. Again, the operator $J_{1}^{\prime}$ is only defined on $\widetilde{\mathcal{H}}_{1}=\mathrm{H}^{1}\left(X_{\varepsilon}\right)$.

The closeness assumptions of Section A.3 now reads as follows:

$$
\begin{gathered}
\left\|J f-J_{1} f\right\|^{2}=\sum_{v \in V} \varepsilon^{-m} \operatorname{vol} U_{\varepsilon, v}|f(v)|^{2} \\
\left\|J^{\prime} u-J_{1}^{\prime} u\right\|^{2}=\sum_{e \in E} \sum_{v \in \partial e} \varepsilon^{m}\left\|\rho_{e}^{ \pm}\right\|_{e}^{2}\left|C_{v} u-N_{e} u(v)\right|^{2} \\
\left|\langle J f, u\rangle-\left\langle f, J^{\prime} u\right\rangle\right| \\
=\left|\sum_{e \in E} \int_{e \times F} \bar{f}(x) u(x, y) \varepsilon^{-m / 2}\left[\mathrm{~d} U_{\varepsilon, e}(x, y)-\varepsilon^{m} \mathrm{~d} F(y) p_{e}(x) \mathrm{d} x\right]\right| \\
\left|\widetilde{\mathfrak{h}}\left(J_{1} f, u\right)-\mathfrak{h}\left(f, J_{1}^{\prime} u\right)\right| \\
=\mid \sum_{e \in E} \int_{e \times F} \bar{f}^{\prime}(x) \partial_{x} u(x, y) \varepsilon^{-m / 2}\left[g_{\varepsilon, e}^{x x} \mathrm{~d} U_{\varepsilon, e}(x, y)-\varepsilon^{m} \mathrm{~d} F(y) p_{e}(x) \mathrm{d} x\right] \\
\| \sum_{e \in E} \sum_{v \in \partial e} \varepsilon^{-m / 2}\left(C_{v} u-N_{e} u(v)\right)\left\langle f_{e}^{\prime},\left(\rho_{e}^{ \pm}\right)^{\prime}\right\rangle_{e} \mid \\
\|J f\|^{2}=\sum_{e \in E} \int_{e \times F}|f(x)|^{2} \varepsilon^{-m} \mathrm{~d} U_{\varepsilon, e}(x, y) \\
\left\|J^{\prime} u\right\|^{2} \leq \sum_{e \times F}|u(x, y)|^{2} \varepsilon^{m} \mathrm{~d} F(y) p_{e}(x) \mathrm{d} x
\end{gathered}
$$

Here, the sign in $\rho_{e}^{ \pm}$is used according to $v=\partial_{ \pm} e$. Note that $J^{\prime} J f=f$. From the RHS we can deduce the necessary assumptions given precisely in the next section. For example, from (2.12) it follows that we must have vol $U_{\varepsilon, v}=o\left(\varepsilon^{m}\right)$, and from (2.13) and (2.14) we see that $g_{\varepsilon, e}$ must be close to a product metric on $U_{e}=e \times F$.

2.4. Assumptions on the graph. We precise here the necessary assumptions in order to estimate the RHS of (2.12) -(2.16) and (2.161). For the graph data we require that the degree is uniformly bounded, i.e., that there exists $d_{0} \in \mathbb{N}$ such that

$$
\operatorname{deg} v \leq d_{0}, \quad v \in V .
$$

We assume in addition that there is a uniform lower bound on the set of length, i.e., there exists $\ell_{0}>0$ (without loss of generality $\ell_{0} \leq 1$ ) such that

$$
\ell_{e} \geq \ell_{0} \quad \text { for all } e \in E \text {. }
$$


We assume that the density function $p_{e}$ is uniformly bounded, i.e., there exist constants $p_{ \pm}>0$ such that

$$
\begin{aligned}
& p_{-} \leq p_{e}(x), \quad \operatorname{dist}\left(x, \partial_{ \pm} e\right) \leq \min \left\{1, \ell_{e} / 2\right\}, \quad e \in E \\
& p_{e}(x) \leq p_{+}, \quad x \in e, \quad e \in E
\end{aligned}
$$

Since $r_{e}(x):=p_{e}(x)^{1 / m}$ will correspond to the radius of the cross section of $U_{\varepsilon, e}$ at $x \in e$, we also denote $r_{ \pm}:=p_{ \pm}^{1 / m}$ the maximal $/$ minimal radius. We want to stress that we allow a sequence of edges $e_{n}$ such that $\ell_{e_{n}} \rightarrow \infty$ or even external edges (i.e., edges with infinite length). In both cases, we do not impose a global lower bound on the density function $p_{e}=r_{e}^{m}$. E.g., a horn-like shape of radius $r_{e}(x)=r_{e, 0} x^{-\beta}$ for the associated edge neighbourhood $U_{\varepsilon, e}$ is allowed for an external edge $e$ (cf. also Remark 3.3).

Definition 2.2. A uniform weighted metric graph is a weighted metric graph $X_{0}=(V, E, \partial, \ell, p)$ satisfying (G1) - G33).

From these assumptions we conclude the following estimates:

Lemma 2.3. Suppose that $(a(v))_{v \in V}$ is a family of non-negative numbers. Then

$$
\sum_{e \in E} \sum_{v \in \partial e} a(v)=\sum_{e \in E}\left(a\left(\partial_{+} e\right)+a\left(\partial_{-} e\right)\right)=\sum_{v \in V}(\operatorname{deg} v) a(v) \leq d_{0} \sum_{v \in V} a(v)
$$

due to (G1). Furthermore,

$$
\sum_{v \in V} \sum_{e \in E_{v}} b_{e}=2 \sum_{e \in E} b_{e}
$$

for a family $\left(b_{e}\right)_{e \in E}$.

Proof. Inequality (2.17) is clear, and from the disjoint union

$$
E=\biguplus_{v \in V} E_{v}^{+}=\biguplus_{v \in V} E_{v}^{-}
$$

the second equality follows immediately.

The next lemma is needed in order to estimate $f(v)$ :

Lemma 2.4. We have

$$
\sum_{v \in V}|f(v)|^{2} \leq \frac{4}{\ell_{0} p_{-}}\|f\|_{1}^{2}
$$

for all $f \in \mathcal{H}_{1}=\mathrm{H}^{1}\left(X_{0}\right)$.

Proof. The estimate follows easily from

$$
|f(0)|^{2} \leq \frac{2}{p_{-}} \max \left\{\frac{1}{\ell}, \ell\right\} \int_{0}^{\ell}\left(|f(x)|^{2}+\left|f^{\prime}(x)\right|^{2}\right) p(x) \mathrm{d} x
$$

where $f \in \mathrm{H}^{1}(0, \ell)$ and $p_{-}:=\inf _{0 \leq x \leq \ell} p(x)$ (cf. [Ku04, Lemma 8]) applied to $\ell:=\min \left\{1, \ell_{e} / 2\right\}$ together with (G2) and (G3).

Finally, we can estimate the cut-off function $\rho_{e}^{ \pm}$using (G2) and (G3): 
Lemma 2.5. The estimate

$$
\left\|\rho_{e}^{ \pm}\right\|_{e}^{2} \leq p_{+} \quad \text { and } \quad\left\|\left(\rho_{e}^{ \pm}\right)^{\prime}\right\|_{e}^{2} \leq \frac{2 p_{+}}{\ell_{0}}
$$

holds for all $e \in E$.

2.5. Assumptions on the manifold. Guided by the classical example of an embedded graph (cf. Section 3.1 and (3.1) ) we assume that the metric $g_{\varepsilon, e}$ on the edge neighbourhood $U_{e}=e \times F$ is given as a perturbation of the product metric

$$
\bar{g}_{\varepsilon, e}:=\mathrm{d} x^{2}+\varepsilon^{2} r_{e}^{2}(x) h(y), \quad(x, y) \in U_{e}=e \times F
$$

with

$$
r_{e}(x):=\left(p_{e}(x)\right)^{1 / m}
$$

where $h$ is the fixed metric on $F, m=\operatorname{dim} F=d-1$ and $p_{e}$ is the density function of the metric graph on the edge $e$.

We denote by $G_{\varepsilon, e}$ and $\bar{G}_{\varepsilon, e}$ the $d \times d$-matrices associated to the metrics $g_{\varepsilon, e}$ and $\bar{g}_{\varepsilon, e}$ with respect to the coordinates $(x, y)$ and assume that the two metrics coincide up to an error term as $\varepsilon \rightarrow 0$, more specifically

$$
G_{\varepsilon, e}=\bar{G}_{\varepsilon, e}+\left(\begin{array}{cc}
o(1) & o(\varepsilon) r_{e} \\
o(\varepsilon) r_{e} & o\left(\varepsilon^{2}\right) r_{e}^{2}
\end{array}\right)=\left(\begin{array}{cc}
1+o(1) & o(\varepsilon) r_{e} \\
o(\varepsilon) r_{e} & \left(\varepsilon^{2} H+o\left(\varepsilon^{2}\right)\right) r_{e}^{2}
\end{array}\right)
$$

uniformly on $U_{e}$. We also assume that these error estimates are uniform in $e$, i.e., that $o\left(\varepsilon^{i}\right)$ does not depend on the edge $e \in E$. As in [EP05, Lemma 4.3] (replacing $\varepsilon$ by $\varepsilon r_{e} \leq \varepsilon r_{+}$) we can show the following estimates

$$
\begin{gathered}
\mathrm{d} U_{\varepsilon, e}(x, y)=\left(1+o_{1}(1)\right) \varepsilon^{m} \mathrm{~d} F(y) p_{e}(x) \mathrm{d} x \\
g_{\varepsilon, e}^{x x}:=\left(G_{\varepsilon, e}^{-1}\right)_{x x}=1+o_{2}(1) \\
\left|\mathrm{d}_{x} u\right|^{2} \leq O_{3}(1)|\mathrm{d} u|_{g_{\varepsilon, e}}^{2} \\
\left|\mathrm{~d}_{F} u\right|_{h}^{2} \leq o_{4}(\varepsilon)|\mathrm{d} u|_{g_{\varepsilon, e}}^{2}
\end{gathered}
$$

where $\mathrm{d}_{x}$ and $\mathrm{d}_{F}$ are the (exterior) derivatives with respect to $x \in e$ and $y \in F$, respectively. Here, $o_{1}(1)$ and $o_{2}(1)$ depend only on $o\left(\varepsilon^{j}\right)$ in (G4) whereas $O_{3}(1)$ and $o_{4}(1)$ depend also on $r_{+}$. The index $i$ in $o_{i}(\cdot)$ is added in order to trace the error estimates in the formulas below. All the estimates are uniform on $U_{e}$ and uniform in $e \in E$ as $\varepsilon \rightarrow 0$.

On the vertex neighbourhood $U_{v}$ we assume that the metric $g_{\varepsilon, v}$ satisfies

$$
c_{-} \varepsilon^{2} g_{v} \leq g_{\varepsilon, v} \leq c_{+} \varepsilon^{2 \alpha} g_{v}
$$

in the sense that there are constants $c_{-}, c_{+}>0$ independent of $v$ such that

$$
c_{-} \varepsilon^{2} g_{v}(z)(w, w) \leq g_{\varepsilon, v}(z)(w, w) \leq c_{+} \varepsilon^{2 \alpha} g_{v}(z)(w, w)
$$

for all $w \in T_{z} U_{v}$ and all $z \in U_{v}$ where $g_{v}$ is the metric $g_{\varepsilon, v}$ with $\varepsilon=1$. The number $\alpha$ in the exponent is assumed to satisfy the inequalities

$$
\frac{d-1}{d}<\alpha \leq 1
$$

In addition, we assume that

$$
c_{\mathrm{vol}}:=\sup _{v \in V} \operatorname{vol} U_{v}<\infty \quad \text { and } \quad \lambda_{2}:=\inf \lambda_{2}^{\mathrm{N}}\left(U_{v}\right)>0
$$


where $\lambda_{2}^{\mathrm{N}}\left(U_{v}\right)$ denotes the second (i.e., the first non-zero) Neumann eigenvalue of $\Delta_{U_{v}}$.

Definition 2.6. A family of graph-like manifolds $X_{\varepsilon}$ w.r.t. the uniform metric graph $X_{0}$ will be called uniform if (G4)-(G7) are satisfied.

We will discuss several examples of uniform metric graphs and graph-like manifolds in Section 3. Let us just finish this subsection with a few comments on the assumptions.

Remark 2.7. (1) The condition (G4) is motivated by the classical example of a curved edge embedded in $\mathbb{R}^{2}$, cf. Section 3.1 and (3.1).

(2) We have assumed an upper bound of the density $p_{e}$ (i.e., the radius function $r_{e}$ ) on the whole edge in (G3). A careful analysis of the proof of (2.22) - 2.25) shows that the first two estimates remain globally true even if $p_{e}$ has no global bound $p_{+}$on $e$. But the last two estimates need the global bound $p_{e}(x) \leq p_{+}$for all $x \in e$ and $e \in E$. Therefore, an infinite edge cannot have a neighbourhood $U_{\varepsilon, e}$ with growing radius $r_{e}(x) \rightarrow \infty$ such as a conical end. It is also forbidden that a sequence of edges $e_{n}$ has neighbourhoods with radius functions $r_{e_{n}}$ unbounded in $n$.

(3) Note that the upper estimate in assumption (G5) does not apply to points at the border of $\partial U_{v}$, since we still assume that $g_{\varepsilon, v}$ is the restriction of a global metric $g_{\varepsilon}$ on $X_{\varepsilon}$; and the cross-section on $U_{\varepsilon, e}$ has a metric of order $O\left(\varepsilon^{2}\right) h$. Nevertheless it is not excluded that $g_{\varepsilon, v}$ scales differently away from $\partial U_{v}$ (for a detailed discussion of such scalings cf. [EP05, Sec. 6]).

(4) The reason for the critical exponent $(d-1) / d$ in (G6) is roughly the following: If $\alpha$ satisfies (G6) then vol $U_{\varepsilon, v} \leq O\left(\varepsilon^{\alpha d}\right)$ decays faster than $\operatorname{vol} U_{\varepsilon, e}=O\left(\varepsilon^{d-1}\right)$. Other decay rates are discussed in [KuZ03, EP05].

(5) Assumption (G7) roughly assures that $U_{v}$ remains small (as family in $v \in V)$ : Suppose that there is an infinite sequence $\left(v_{n}\right) \subset V$ such that $U_{v_{n}}=U_{v_{0}}$ as sets and that $g_{v_{n}}=\rho_{n}^{2} g$ for a sequence $\rho_{n} \rightarrow \infty$ of positive numbers. This behaviour does not contradict (G5) since (G5) is only a relative bound w.r.t. a fixed metric $g_{v}$. But (G7) is no longer satisfied.

In addition, the eigenvalue estimate assures that $U_{\varepsilon, v}$ as well as $\left(U_{v_{n}}\right)_{n}$ for a sequence $\left(v_{n}\right)_{n} \subset V$ do not separate into two (or more) parts as $\varepsilon \rightarrow 0$ or $n \rightarrow \infty$, respectively. This could happen e.g. if $U_{\varepsilon, v}$ or $\left(U_{v_{n}}\right)_{n}$ consists of two (or more) large parts joined by small cylinders which shrink as $\varepsilon \rightarrow 0$ resp. $n \rightarrow \infty$. This could lead to a decoupling of the edges emanating from such a vertex or such a sequence of vertices.

(6) We want to stress that $U_{v}$ cannot either become too small as $v \rightarrow \infty$ for some sequence of vertices. This is at first sight not obvious, since a priori, (G5) is not a restriction of $g_{v}$ as family in $v \in V$ and (G7) roughly says that $U_{v}$ remains small as family in $v$. But one has to take into account that $g_{\varepsilon, e}$ and $g_{\varepsilon, v}$ are restrictions of a global smooth metric $g_{\varepsilon}$. In particular, the metrics on the common boundary $\partial_{e} U_{v}$ of $U_{e}$ and $U_{v}$ must be the same; and therefore, the uniform estimates of $g_{\varepsilon, e}$ in (G4) become uniform estimates of $g_{\varepsilon, v}$ (take e.g. a sequence $\rho_{n} \rightarrow 0$ and argue as in the previous remark). 
Remark 2.8. Without loss of generality we can assume that $\partial_{e} U_{v}$ has a collar neighbourhood $U_{e, v}=\left(0, \ell_{-}\right) \times F$ in which the metric $g_{v}$ on $U_{v}$ has the form

$$
g_{e, v}=\mathrm{d} \bar{x}^{2}+h_{\bar{x}}
$$

for $(\bar{x}, y) \in U_{e, v}$, i.e., the collar neighbourhood has length $\ell_{-}$for some global constant $0<\ell_{-}<1$ (e.g., $\ell_{-}=\ell_{0} / 2$, cf. also Figure 3 where $\ell_{-}<\ell_{0} / 2$ ). Here, $\partial_{e} U_{v}$ is the part of the boundary (diffeomorphic to $F$ ) where the edge neighbourhood of $e$ meets. In addition, we assume that estimates similar to (2.22) and (2.24) are fulfilled (with $\varepsilon=1, x$ replaced by $\bar{x}$ and $g_{\varepsilon, e}$ replaced by $\left.g_{e, v}\right)$.

If this condition is not satisfied, we just have to modify the decomposition of the manifold into edge and vertex neighbourhoods in order that $U_{\varepsilon, v}$ has at least a cylindrical part of length $\varepsilon \ell_{-}$(taken from the edge neighbourhood). The desired estimates (2.22) and (2.24) follow now from (G4) with the new variable $\bar{x}=x / \varepsilon$.

2.6. Main result. We are now able to prove the following lemmas needed to complete the proof of the closeness assumptions. Mainly, the estimates are already given in EP05] but since there, we only considered compact graphs and compact manifolds, a precise control of the constants was not necessary. We do not repeat the proofs given there but we state the necessary results together with their dependence on the constants given in the uniformity assumptions.

Lemma 2.9. We have

$$
\|u\|_{\partial_{e} U_{v}}^{2} \leq c_{\mathrm{tr}}\left(\|u\|_{U_{v}}^{2}+\|d u\|_{U_{v}}^{2}\right)
$$

for all $u \in \mathrm{H}^{1}\left(U_{v}\right)$ where

$$
c_{\mathrm{tr}}:=\frac{2 p_{+}\left(1+o_{1}(1)\right)\left(1+O_{3}(1)\right)}{p_{-}\left(1-o_{1}(1)\right) \ell_{-}} .
$$

Proof. The estimate is an immediate consequence of (2.19) and Remark 2.8.

The following lemma roughly states that the transversal average on the boundary $\partial_{e} U_{v}$ is close to the total average on $U_{v}$. The proof is based on the fact that the second Neumann eigenvalue of $U_{\varepsilon, v}$ tends to $\infty$ as $\varepsilon \rightarrow 0$ (cf. [EP05, Lemma 5.5]):

Lemma 2.10. The inequality

$$
\varepsilon^{m}\left|C_{v} u-N_{e} u(v)\right|^{2} \leq \widetilde{c}_{\mathrm{tr}} \varepsilon^{2 \alpha-1}\|d u\|_{U_{\varepsilon, v}}^{2}
$$

holds for all functions $u \in \mathrm{H}^{1}\left(U_{\varepsilon, v}\right)$ and $v=\partial_{ \pm} e$ where

$$
\widetilde{c}_{\mathrm{tr}}:=\frac{c_{-}^{2}}{p_{-}\left(1-o_{1}(1)\right) c_{+}^{d}}\left(\frac{1}{\lambda_{2}}+1\right) c_{\mathrm{tr}} .
$$

Note that $\varepsilon^{2 \alpha-1} \rightarrow 0$ as $\varepsilon \rightarrow 0$ since $\alpha>(d-1) / d \geq 1 / 2$.

The next lemma assures that higher transversal modes does not contribute too much (cf. EP05, Lemmas 3.1 and 4.4]). Essentially, it is the observation, that $N_{e} u-u$ is the projection onto the orthogonal complement of the first (constant) eigenfunction on $F$ : 
Lemma 2.11. We have

$$
\left\|N_{e} u-u\right\|_{U_{\varepsilon, e}}^{2} \leq c_{\mathrm{ed}} O_{4}(\varepsilon)\|d u\|_{U_{\varepsilon, e}}^{2}
$$

for all $u \in \mathrm{H}^{1}\left(U_{\varepsilon, e}\right)$ where

$$
c_{\mathrm{ed}}:=\frac{\left(1+o_{1}(1)\right)}{\left(1-o_{1}(1)\right) \lambda_{2}(F)} .
$$

Finally, we need to assure that there is no concentration at the vertex neighbourhoods in any bounded spectral interval (cf. [EP05, Corollary 5.8]):

Lemma 2.12. The estimate

$$
\|u\|_{U_{\varepsilon, v}}^{2} \leq c_{\mathrm{vX}} \varepsilon^{\alpha d-m}\|u\|_{1, \widehat{U}_{\varepsilon, v}}^{2}
$$

holds for all $u$ in $\mathrm{H}^{1}\left(\widehat{U}_{\varepsilon, v}\right)$ where $\widehat{U}_{\varepsilon, v}:=U_{\varepsilon, v} \cup \bigcup_{e \in E_{v}} U_{\varepsilon, e}$ and $c_{\mathrm{vx}}$ depends only on $\ell_{0}, p_{ \pm}, o_{1}(1), O_{3}(1), c_{ \pm}, c_{\mathrm{vol}}, \lambda_{2}$ and $\widetilde{c}_{\mathrm{tr}}$.

We are now able to estimate the RHS of the closeness assumptions (2.12)-(2.16) and (2.16]). For the first one, we have

$$
\left\|J f-J_{1} f\right\|^{2} \leq \frac{4 c_{+}^{d / 2} c_{\mathrm{vol}}}{\ell_{0} p_{-}} \varepsilon^{\alpha d-m}\|f\|_{1}^{2}
$$

for $f \in \mathcal{H}_{1}$ using Lemma 2.4 and (G5). Note that $\varepsilon^{\alpha d-m} \rightarrow 0$ as $\varepsilon \rightarrow 0$ due to (G6). Next, we have

$$
\left\|J^{\prime} u-J_{1}^{\prime} u\right\|^{2} \leq p_{+} \tilde{c}_{\mathrm{tr}} \varepsilon^{2 \alpha-1} \sum_{e \in E} \sum_{v \in \partial e}\|d u\|_{U_{\varepsilon, v}}^{2} \leq d_{0} p_{+} \tilde{c}_{\mathrm{tr}} \varepsilon^{2 \alpha-1} \widetilde{\mathfrak{h}}(u)
$$

using Lemma 2.10, Lemma 2.5 and Equation (2.17). The estimation of (2.13), i.e.,

$$
\left|\langle J f, u\rangle-\left\langle f, J^{\prime} u\right\rangle\right| \leq o_{1}(1)\|f\|\|u\|
$$

follows from (2.22). Similarly, the estimate (2.14) can be proven by

$$
\left|\widetilde{\mathfrak{h}}\left(J_{1} f, u\right)-\mathfrak{h}\left(f, J_{1}^{\prime} u\right)\right| \leq\left(o(1)+\left[\frac{2 d_{0} p_{+} \widetilde{c}_{\mathrm{tr}}}{\ell_{0}}\right]^{1 / 2} \varepsilon^{\alpha-1 / 2}\right) \mathfrak{h}(f)^{1 / 2} \widetilde{\mathfrak{h}}(u)^{1 / 2}
$$

where $o(1)$ depends on the errors in (G4). Again, we have used Lemma 2.10, Lemma 2.5 and Equation (2.17). Estimate (2.15) follows from

$$
\begin{aligned}
\left\|J J^{\prime} u-u\right\|^{2}= & \sum_{e \in E}\left\|N_{e} u-u\right\|_{U_{\varepsilon, e}}^{2}+\sum_{v \in V}\|u\|_{U_{\varepsilon, v}}^{2} \\
& \leq c_{\mathrm{ed}} O_{4}(\varepsilon) \sum_{e \in E}\|d u\|_{U_{\varepsilon, e}}^{2}+c_{\mathrm{vx}} \varepsilon^{\alpha d-m} \sum_{v \in V}\|u\|_{1, \widehat{U}_{\varepsilon, v}}^{2} \\
& \leq 3\left(c_{\mathrm{ed}} o_{4}(\varepsilon)+c_{\mathrm{vx}} \varepsilon^{\alpha d-m}\right)\|u\|_{1}^{2}
\end{aligned}
$$

where we also used (2.18) in the second estimate. Finally, Assumption (A.13) follows from

$$
\|J f\|^{2} \leq\left(1+o_{1}(1)\|f\|^{2} \quad \text { and } \quad\left\|J^{\prime} u\right\|^{2} \leq \frac{1}{1-o_{1}(1)}\|u\|^{2}\right.
$$

and (2.22). We therefore have proven 
Theorem 2.13. Suppose that the metric graph $X_{0}$ and the family of graph-like manifolds $X_{\varepsilon}$ is given as below and satisfy the uniformity conditions (G1) -(G7). Then the generalised weighted Neumann Laplacian on the graph $\left(\Delta_{X_{0}}, \mathrm{~L}_{2}\left(X_{0}\right)\right)$ and the (Neumann) Laplacian on the manifold $\left(\Delta_{X_{\varepsilon}}, L_{2}\left(X_{\varepsilon}\right)\right)$ are $\delta$-close of order 1 where $\delta=o(1)$ as $\varepsilon \rightarrow 0$. In particular, all the results of Appendix $\mathbb{A}$ are true, e.g., the convergence of eigenfunctions stated in Theorem A.19 or the spectral convergence in Theorem A.13.

In particular, we have the following consequence of the convergence results of Appendix $\mathrm{A}$

Remark 2.14. Due to Theorem A.10 and Thms. A.7 A.8 we can approximate the complicated operator on the manifold $\varphi\left(\Delta_{X_{\varepsilon}}\right)$ by the simpler operator $J \varphi\left(\Delta_{X_{0}}\right) J^{\prime}$ up to an error, where e.g. $\varphi(\lambda)=(\lambda+1)^{-1}$ (resolvent), $\varphi_{t}(\lambda)=\mathrm{e}^{-t \lambda}$ (heat operator) or $\varphi=\mathbb{1}_{I}$ (spectral projection). Saito [Sa00] obtained a similar but weaker assertion in the resolvent case.

\section{EXAmples AND APPlications of THE SPECTRAL CONVERGENCE}

In this section we give several classes of examples for uniform graphs and manifolds. We also provide consequences of the above-mentioned spectral convergence.

3.1. Embedded graphs and graph-like manifolds. Let us start with an embedded metric graph as an explicite example in order to illustrate the geometric meaning of the uniformity assumptions (G1)-G7). This situation has originally been treated in KuZ01, RS01] on compact metric graphs (cf. also EP05, Ex. 4.2])), and on trees in [Sa00:

Suppose that the weighted metric graph $X_{0}=(V, E, \partial, \ell, p)$ is isometrically embedded in $\mathbb{R}^{2}$ via the maps $\psi_{e}:\left(0, \ell_{e}\right) \longrightarrow \mathbb{R}^{2}$. Then

$$
\Psi_{\varepsilon, e}(x, y):=\psi_{e}(x)+\varepsilon r_{e}(x) y n_{e}(x), \quad(x, y) \in(0, \ell) \times[-1 / 2,1 / 2] \cong e \times F
$$

defines a weighted tubular neighbourhood of the edge $e$ considered as subset of $\mathbb{R}^{2}$. Here, $n_{e}$ is a unit vector field normal to the tangent vector field $\dot{\psi}_{e}$ and $r_{e}(x)$ defines the radius of the neighbourhood. Let $X_{\varepsilon}$ be the union of the closures of the range of all $\Psi_{\varepsilon, e}, e \in E$. Denote by

$$
\kappa_{e}:=\dot{\psi}_{1} \ddot{\psi}_{2}-\ddot{\psi}_{1} \dot{\psi}_{2}
$$

the signed curvature of the embedded edge $e$. We assume that there are global constants $\ell_{0}, \beta_{0}, \kappa_{0}, r_{ \pm}, \dot{r}_{0}>0$ such that

$$
\begin{array}{rlrl}
\measuredangle\left(e, e^{\prime}\right) & \geq \beta_{0}, & \tan \left(\beta_{0} / 2\right) & >r_{+} / \ell_{0} \\
\left|\kappa_{e}(x)\right| & \leq \kappa_{0}, \quad x \in e \\
\ell_{e} & \geq \ell_{0}, & r_{e}(x) & \geq r_{-}, \quad d(x, \partial e) \leq \min \left\{1, \ell_{e} / 2\right\} \\
\left|\dot{r}_{e}(x)\right| & \leq \dot{r}_{0}, & r_{e}(x) & \leq r_{+}, \quad x \in e .
\end{array}
$$

Here, $\measuredangle\left(e, e^{\prime}\right)$ denotes the angle between the tangent vectors $\dot{\psi}_{e}$ and $\dot{\psi}_{e^{\prime}}$ of the embedded edges at the vertex $v$ for $e, e^{\prime} \in E_{v}, e \neq e^{\prime}$. 
We claim that under these assumptions, $X_{\varepsilon}$ is a uniform family of graphlike manifolds ${ }^{2}$ embedded in $\mathbb{R}^{2}$ associated to the uniform metric graph $X_{0}$ with weights $p_{e}:=r_{e}$ :

Theorem 3.1. Under the assumptions (E1)-(E4) the metric graph $X_{0}$ and the associated weighted neighbourhood $X_{\varepsilon}$ satisfy (G1)-(G7) for $\varepsilon$ small enough. In particular, the generalised weighted Neumann Laplacian on the graph $\left(\Delta_{X_{0}}, \mathrm{~L}_{2}\left(X_{0}\right)\right)$ and the Neumann Laplacian $\left(\Delta_{X_{\varepsilon}}^{\mathrm{N}}, \mathrm{L}_{2}\left(X_{\varepsilon}\right)\right)$ are $\delta$-close of order 1 where $\delta=O\left(\varepsilon^{1 / 2}\right)$ as $\varepsilon \rightarrow 0$, and therefore, the convergence results of Appendix $A$ are true.

Proof. The bounded degree assumption (G1) follows from (E1) with $d_{0}=\left\lceil 2 \pi / \beta_{0}\right\rceil$. (G2) and (G3) are clear. We decompose $X_{\varepsilon}$ into the edge and vertex neighbourhoods $U_{\varepsilon, e}$ and $U_{\varepsilon, v}$, resp., in the way that

$$
U_{\varepsilon, e}=\Psi_{\varepsilon, e}\left(\left(\varepsilon \ell_{0} / 2, \ell_{e}-\varepsilon \ell_{0} / 2\right) \times F\right)
$$

denotes the edge neighbourhood of $e$ shortened by the amount $\varepsilon \ell_{0}$ belonging to the vertex neighbourhoods of $\partial_{-} e$ and $\partial_{+} e$. A straightforward calculation now shows that the metric of $U_{\varepsilon, e} \cong\left(e \times F, g_{\varepsilon}\right)$ is represented by

$$
G_{\varepsilon}=\left(\begin{array}{cc}
{\left[\left(1+\varepsilon \kappa_{e} r_{e} y\right)^{2}+\varepsilon^{2} y^{2} \dot{r}_{e}^{2}\right]\left(1-\varepsilon \ell_{0} / \ell_{e}\right)^{2}} & \varepsilon^{2} r_{e} \dot{r}_{e} y\left(1-\varepsilon \ell_{0} / \ell_{e}\right) \\
\varepsilon^{2} r_{e} \dot{r}_{e} y\left(1-\varepsilon \ell_{0} / \ell_{e}\right) & \varepsilon^{2} r_{e}^{2}
\end{array}\right) .
$$

The uniformity condition (G4) is fulfilled due to the curvature assumption (E2) and due to (E4) and we obtain

$$
\begin{aligned}
o_{1}(1) & =2 r_{+} \varepsilon\left(1+\varepsilon \kappa_{0} r_{+}\right)^{1 / 2}, & 1+o_{2}(1) & =1+\varepsilon C\left(\kappa_{0}, r_{+}\right), \\
O_{3}(1) & =C\left(\kappa_{0}, r_{+}, \dot{r}_{0}\right), & o_{4}(\varepsilon) & =2 r_{+}^{2} \varepsilon^{2}
\end{aligned}
$$

provided $0<\varepsilon<\varepsilon_{0}=\varepsilon_{0}\left(\kappa_{0}, r_{+}, \dot{r}_{0}\right)$, where $C(\cdot)$ depend only on the indicated constants, since, e.g.,

$$
\operatorname{det} G_{\varepsilon}^{1 / 2}=\varepsilon r_{e}\left(1+\varepsilon \ell_{0} / \ell_{e}\right)\left|1+\varepsilon \kappa_{e} r_{e} y\right| .
$$

A similar calculation on $U_{\varepsilon, v} \cap \Psi_{\varepsilon, e(e \times F)}, v \in \partial e$, now using $\bar{x}=x / \varepsilon$ and $y \in F$ as coordinates (as far as possible on $U_{\varepsilon, v}$ ) shows that (G5) and (G6) are satisfied with $\alpha=1$ and $c_{ \pm}$depending only on $\kappa_{0}, r_{ \pm}$and $\dot{r}_{0}$. As unscaled set $U_{v}$ we use the "straightened" version of $\frac{1}{\varepsilon} U_{\varepsilon, v}$, i.e., we replace the edge parts in $\frac{1}{\varepsilon} U_{v, \varepsilon}$ by their tangentials at $v$; the tubular neighbourhood has width $r_{e}(0)$ (cf. Figure 3).

Next, the volume estimate of (G7) is satisfied due to the angle assumption (E1):

$$
\operatorname{vol} U_{v} \leq \sum_{e \in E_{v}} r_{e}(v) \ell_{0} / 2 \leq d_{0} r_{+} \ell_{0} / 2
$$

Due to (E1) we can place at each end of $U_{v}$ a rectangle (denoted in dark grey in Figure 3) of length $\ell_{-}=\left(\ell_{0}-r_{+} \cot \left(\beta_{0} / 2\right)\right) / 2>0$ and width $r_{e}(0) \in\left[r_{-}, r_{+}\right]$. These rectangles are the collar neighbourhoods mentioned in Remark 2.8. The Neumann eigenvalues depend continuously on the angles $\measuredangle\left(e, e^{\prime}\right) \geq \beta_{0}$ and on the widths $r_{e}(0) \in\left[r_{-}, r_{+}\right]$, so (G7) follows.

\footnotetext{
${ }^{2}$ Of course, there are other possibilities how to define $X_{\varepsilon}$ close to the vertices, which still lead to a uniform graph-like manifold. One can e.g. smoothen the singularities at the boundary $\partial U_{\varepsilon, v} \cap \partial X_{\varepsilon}$ (cf. Figure 3). In our case, $\partial X_{\varepsilon}$ has singularities but this does not matter in the proof of Theorem 2.13 and Theorem 3.1
} 

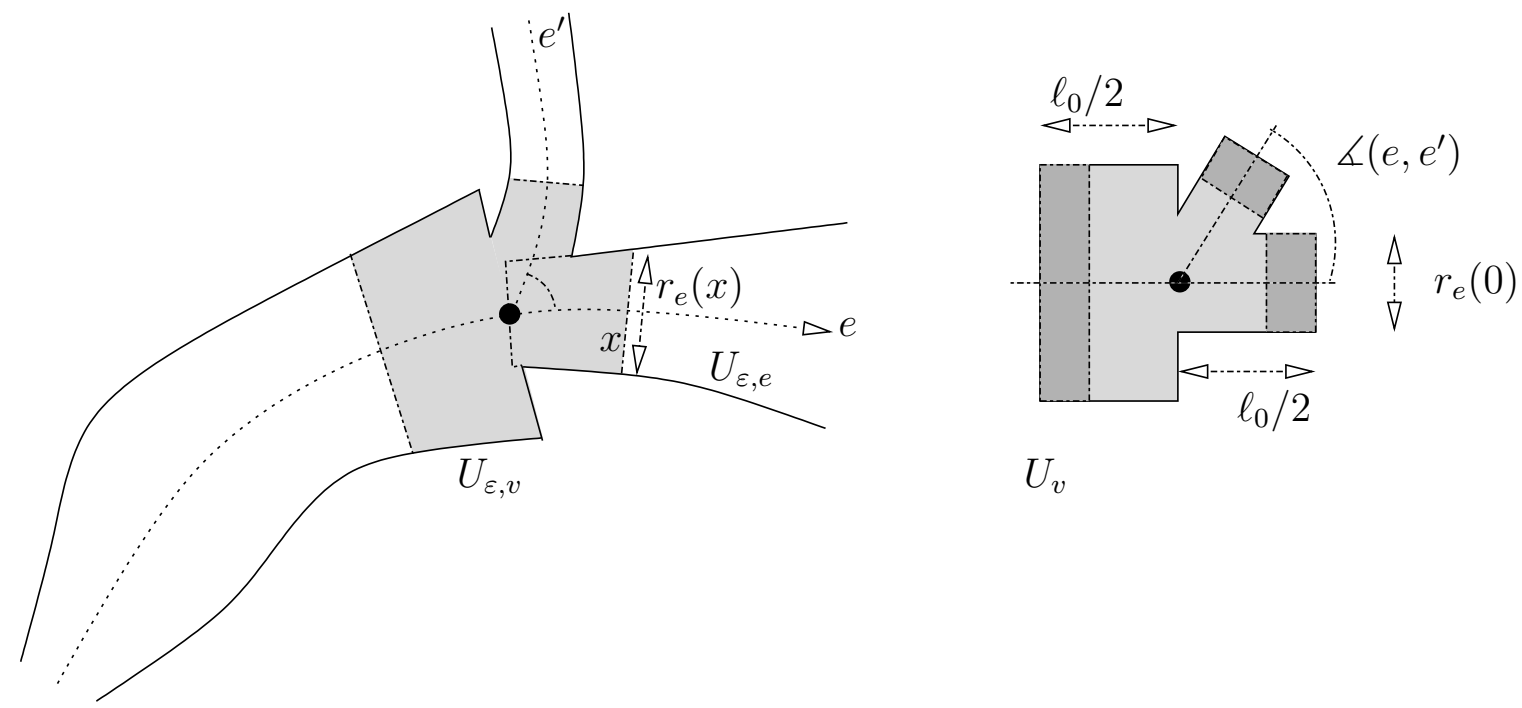

Figure 3. Decomposition of the weighted neighbourhood $X_{\varepsilon}$ and the unscaled vertex neighbourhood $U_{v}$. In dark grey we denoted the collar neighbourhoods mentioned in Remark 2.8. Note that these rectangles exists due to (E1).

Remark 3.2. Although the curvature $\kappa_{e}$ of the edge and the angle $\measuredangle\left(e, e^{\prime}\right)$ between two adjacent edges are not detectable in the limit (at least not in our first order approximation of an eigenvalue $\lambda(\varepsilon)=\lambda(0)+o(1))$, we nevertheless need the uniform assumptions (E1) and (E2). Using the direct eigenvalue estimates of Remark A.16 on compact spaces one can show that $\lambda_{k}(\varepsilon)=\lambda_{k}(0)+O\left(\varepsilon^{1 / 2}\right)$. It would be interesting whether one can detect information on the curvature or the angles between the edges via an asymptotic expansion of $\lambda_{1}(\varepsilon)$. For a curved tubular neighbourhood with Dirichlet boundary conditions around a closed curve of length $\ell$ with positive curvature in $\mathbb{R}^{3}$, the first eigenvalue expands as

$$
\lambda_{1}^{\mathrm{D}}(\varepsilon)=\frac{\lambda_{1}}{\varepsilon^{2}}-\frac{3}{4 L} \int_{0}^{\ell} \kappa_{1}(s)^{2} \mathrm{~d} s+O(\varepsilon)
$$

(cf. KaP88, Thm. 4.1]) where $\lambda_{1}$ is the first Dirichlet eigenvalue of the unit disc and $\kappa_{1}$ is the curvature of the curve.

Remark 3.3. Note that we do not need a global lower bound on the radius $r_{e}$, i.e., infinite edges with a shrinking neighbourhood are allowed (e.g. horn-like shapes as in DaS92. If the spectrum of the Laplacian on the corresponding edge neighbourhood $U_{\varepsilon, e}$ is $[0, \infty)$, our analysis does not give new information. More interesting cases are provided if the spectrum on $U_{\varepsilon, e}$ is purely discrete, e.g. for radial functions decaying fast enough like $r_{e}(x)=\mathrm{e}^{-x^{\beta}}, \beta>1$ (cf. EvHa89, DaS92]). The weighted graph Laplacian on $e$ now has the form $(H f)_{e}=-f_{e}^{\prime \prime}+$ $m \beta x^{\beta-1} f_{e}^{\prime}$. An example of a horn-like end with infinitely many spectral gaps in the essential spectrum was constructed in [Lo01, Thm. 3]. In principle, these results can be recovered with our analysis.

\subsection{Examples constructed from a finite number of building blocks.}


Covering manifolds. An important class of examples satisfying the uniformity conditions are coverings with a compact quotient: Clearly, a covering metric graph $X_{0} \rightarrow M_{0}$ with compact quotient $M_{0}$ is uniform. Similarly, an associated family of graph-like covering manifold $X_{\varepsilon} \rightarrow M_{\varepsilon}$ with compact quotient $M_{\varepsilon}$ is uniform once (G4)-(G6) are fulfilled.

For abelian covering groups (and for some classes of non-abelian groups, cf. [LP04]) the spectral convergence on compact graphs and manifolds would be enough: The Floquet theory allows to describe the spectrum on the covering via a family of Laplacians on the compact quotient. Nevertheless our result here is more general since we can treat an arbitrary covering.

More generally, the assumptions (G4)-G7) are fulfilled if the number of isomorphism classes of $U_{v}$ and $U_{e}$ are finite, i.e., if we construct the graph-like manifold out of a finite number of building blocks as in a plumber's shop. An example is given in Section 3.3 where we construct a graph-like manifold according to the Sierpiński graph (cf. Figure 4). Here, only two different vertex neighbourhood building blocks are necessary. Note that this manifold has a fractal structure, not locally, but globally.

Spectral gaps and eigenvalues in gaps. Typically, operators on coverings have a band-gap type spectrum, i.e., the spectrum is the locally finite union of compact intervals (maybe reduced to a point). The spectral convergence ensures e.g. the existence of spectral gaps as $\varepsilon \rightarrow 0$ once $\sigma\left(\Delta_{X_{0}}\right)$ has spectral gaps and $\varepsilon$ is small enough:

Theorem 3.4. Suppose that $M_{0}$ is a compact graph with associated uniform graph-like manifold $M_{\varepsilon}$. Denote by $X_{0}$ resp. $X_{\varepsilon}$ a covering of $M_{0}$ resp. $X_{\varepsilon}$ such that $X_{\varepsilon}$ is a graph-like manifold associated to $X_{0}$. If the generalised Neumann Laplacian on $X_{0}$ has a spectral gap $(a, b)$, i.e., $\sigma\left(\Delta_{X_{0}}\right) \cap(a, b)=\emptyset$, then the Laplacian $\Delta_{X_{\varepsilon}}$ has a spectral gap close to $(a, b)$ provided $\varepsilon$ is small enough.

Covering metric graphs $X_{0} \rightarrow M_{0}$ with spectral gaps are given e.g. in EP05, Sec. 9.4-9.6]; the simplest example is maybe the Cayley graph of the group $\Gamma=$ $\mathbb{Z} \times \mathbb{Z}_{p}$ where $\mathbb{Z}_{p}$ denotes the cyclic group of order $p$ has a spectral gap iff $p$ is odd. A similar example consists of a regular rooted tree (cf. Theorem [3.7). A different procedure of creating gaps in a metric graph is provided by the socalled graph-decoration. Roughly speaking, the new graph $\hat{X}_{0}$ is obtained from a given infinite graph $X_{0}$ by attaching a fixed (compact) graph $M_{0}$ to each vertex $v$ of $X_{0}$. The Laplacian on $\hat{X}_{0}$ now has spectral gaps. This result has been established for a discrete graph in [AS00]. The general case for quantum graphs is announced in $\mathrm{Ku} 05$ and proved in the case when $X_{0}$ is a compact graph (in the sense that there is no spectrum of the decorated graph near (certain parts) of the spectrum of the decorating graph). For quantum graphs, there are related examples leading to gaps (cf. [AEL94, [E95]). A similar effect by attaching a single loop to each vertex of a periodic graph has been used in [EP05, Sec. 9]. The case of periodically arranged manifolds connected by line sements or through points has been analysed in BEG03, BGL05.

Finally, another class of examples is given by fractal metric graphs, i.e., graphs arranged in a self-similar manner (cf. Theorem 3.10). The main point here is that the metric graph spectrum has a fractal structure, so once, $\varepsilon$ is small enough, the 
corresponding Laplacian on a graph-like manifold has an arbitrary (but a priori finite) number of spectral gaps in the compact spectral interval $[0, \Lambda]$.

Eigenvalues in gaps. Suppose that $X_{0}$ is a uniform metric graph such that its generalised Neumann Laplacian has a spectral gap, namely, $\sigma\left(\Delta_{X_{0}}\right) \cap(a, b)=\emptyset$. By the previous example, a corresponding uniform graph-like manifold $X_{\varepsilon}$ has also a spectral gap close to $(a, b)$ provided $\varepsilon$ is small enough.

Now if we change the metric graph locally, e.g. by attaching a loop of length $\ell_{1}$ at a fixed vertex $v_{1} \in V$ (call the perturbed graph $\hat{X}_{0}$ ) then the generalised Neumann Laplacian on $\hat{X}_{0}$ has additional eigenvalues $\lambda_{k}=\left(2 \pi k / \ell_{1}\right)^{2}$ with eigenfunctions located on the loop and vanishing at $v_{1}$ and on the rest of the graph (maybe there are more additional eigenvalues). For example, if $2 \pi / \sqrt{b}<\ell_{1}<2 \pi / \sqrt{a}$ then the ground state of the loop lies in $(a, b)$. Note that the essential spectrum of $X_{0}$ and the perturbed metric graph $\hat{X}_{0}$ are the same since the perturbation is compact; in particular, only discrete eigenvalues occur in the spectral gap. Due to the spectral convergence Theorem 2.13, a corresponding (uniform) graph-like manifold now must have (at least) an eigenvalue in $(a, b)$. Furthermore, if the corresponding eigenvalue of the quantum graph is simple (i.e., there are no other eigenvalues of the looped graph $\hat{X}_{0}$ at $\left.\lambda_{1}=\left(2 \pi / \ell_{0}\right)^{2}\right)$, then there is a unique eigenvalue close to $\lambda_{1}$ of multiplicity 1 , and the corresponding eigenfunction of the graph-like manifold is close to the eigenfunction of the metric graph in the sense of Theorem A.12 More generally, we have:

Theorem 3.5. Suppose that $\Delta_{X_{0}}$ has a discrete eigenvalue $\lambda$ of multiplicity $m$ and $\sigma\left(\Delta_{X_{0}}\right) \cap I=\{\lambda\}$. Let $X_{\varepsilon}$ be a graph-like manifold associated to $X_{0}$. Then $\Delta_{X_{\varepsilon}}$ has $m$ eigenvalues (not all necessarily distinct) converging to $\lambda$ as $\varepsilon \rightarrow 0$ and the eigenprojections converge in norm, i.e., $\left\|J^{\prime} P_{\varepsilon} J-P_{0}\right\| \rightarrow 0$ as $\varepsilon \rightarrow 0$ where $P_{\varepsilon}$ is the eigenprojection of $\Delta_{X_{\varepsilon}}$ onto the interval $I, \varepsilon \geq 0$.

If in particular, $\lambda$ is a simple eigenvalue with eigenfunction $\varphi$, then there exists an eigenfunction $\varphi_{\varepsilon}$ of $\Delta_{X_{\varepsilon}}$ such that $\left\|J \varphi-\varphi_{\varepsilon}\right\| \rightarrow 0$.

Roughly speaking, the theorem says, that an eigenfunction on the graph-like manifold is approximately given by the corresponding eigenfunction on the graph, extended constantly in the transversal direction (and set to 0 in the vertex neighbourhood).

Eigenvalues in gaps have been discussed e.g. in AADH94, P03a (see also the references therein). One can interprete such a local perturbation as an impurity of a periodic structure, say, a crystal or a semi-conductor. The additional eigenvalue in the gap now corresponds to an additional energy level and a bound state. Note that in $\mathrm{P03a}$ it was assumed that $X_{\varepsilon}$ is a covering manifold and that it fulfilled a gap condition in the sense that there is a fundamental domain $D$ such that $\lambda_{k}^{\mathrm{D}}(D)<\lambda_{k+1}^{\mathrm{N}}(D)$ for some $k$ (Dirichlet and Neumann eigenvalues on $D$ ). This is in general a stronger condition than just the assumption that $\Delta_{X_{\varepsilon}}$ has a spectral gap. Here, we only need to know that the generalised Neumann Laplacian $\Delta_{X_{0}}$ on the graph has a spectral gap which is often easy to show.

3.3. Equilateral metric graphs and discrete graphs. In this subsection we will analyse the special case when all length of the metric graph $X_{0}=(V, E, \partial, \ell)$ are the same, say $\ell_{e}=\ell$. Under these assumptions, there is a nice relation between 
the spectrum of the metric graph Laplacian $\Delta_{X_{0}}$ (with weights $p_{e}=1$ ) acting on $\mathrm{L}_{2}\left(X_{0}\right)=\oplus_{e} \mathrm{~L}_{2}(0, \ell)$ and the discrete Laplacian $\Delta_{G}$ of the graph $G=(V, E, \partial)$. Roughly speaking, the spectrum of $\Delta_{X_{0}}$ consists of infinitely many (distorted) copies of the spectrum of $\Delta_{G}$ arranged in a row. This relation allows us to profit from the vast literature on $\Delta_{G}$ and to carry over calculations of spectra of the discrete to the continuous graph Laplacian.

The discrete Laplacian is given by

$$
\left(\Delta_{G} a\right)(v):=-\frac{1}{\operatorname{deg} v} \sum_{w \sim v}(a(w)-a(v)), \quad a \in \ell_{2}(V)
$$

where $w \sim v$ means that $v$ and $w$ are joined by an edge. The discrete Laplacian acts in the weighted space $\ell_{2}(V)$ consisting of all sequences $a=\left(a_{v}\right)_{v}$ with finite weighted norm

$$
\|a\|_{\ell_{2}(V)}:=\sum_{v \in V} \operatorname{deg} v|a(v)|^{2} .
$$

This operator is bounded and has spectrum in $[0,2]$.

In Ca97 one can find the following nice relation between the spectrum of the generalised Neumann Laplacian $\Delta_{X_{0}}$ and the discrete Laplacian $\Delta_{G}$. The Dirichlet spectrum $\Sigma^{\mathrm{D}}=\left\{(k \pi / \ell)^{2} \mid k \in \mathbb{N}\right\}$ of an individual edge $e \cong(0, \ell)$ always plays a special role. Since we are only interested in qualitative results (e.g. the fractal example in Theorem [3.10) we exclude the Dirichlet spectrum here. A more detailed discussion on the Dirichlet spectrum can be found in Ku05] and [Ca97]. We set $g(\lambda)=1-\cos (\ell \sqrt{\lambda})$. Note that $\Sigma^{\mathrm{D}}=g^{-1}\{0,2\}$.

Theorem 3.6. Assume that $G=(V, E, \partial)$ is a countable, connected graph with $\operatorname{deg} v \in\left\{2,3, \ldots, d_{0}\right\}, v \in V$, and without self-loops then

$$
\sigma_{\bullet}\left(\Delta_{X_{0}}\right) \backslash \Sigma^{\mathrm{D}}=g^{-1}\left(\sigma_{\bullet}\left(\Delta_{G}\right) \backslash\{0,2\}\right),
$$

i.e., for $\lambda \neq \Sigma^{\mathrm{D}}$, we have $\lambda \in \sigma_{\bullet}\left(\Delta_{X_{0}}\right)$ iff $g(\lambda) \in \sigma_{\bullet}\left(\Delta_{G}\right)$. Here $\bullet \in$ $\{\mathrm{p}, \mathrm{c}, \emptyset, \mathrm{pp}$, disc, ess $\}$ denotes either the point spectrum (the set of eigenvalues $\sigma_{\mathrm{p}}$ ), the complement of the eigenvalues ${ }^{3}\left(\sigma_{\mathrm{c}}=\sigma \backslash \sigma_{\mathrm{p}}\right)$, the entire spectrum $(\sigma)$, the pure point spectrum (the closure of the set of eigenvalues, i.e., $\sigma_{\mathrm{pp}}=\overline{\sigma_{\mathrm{p}}}$ ), the discrete or the essential spectrum. Furthermore, the eigenvalue $\lambda$ of $\Delta_{X_{0}}$ has multiplicity $m$ iff the eigenvalue $g(\lambda)$ of $\Delta_{G}$ has multiplicity $m$.

Proof. The assertion has been proved for the point spectrum and its complement in [Ca97] and therefore also for the entire spectrum. Since $g$ is a local homeomorphism on the complement of $\Sigma^{\mathrm{D}}$, the statement on $\sigma_{\mathrm{pp}}$ also follows. In addition, note that

$$
\begin{gathered}
U_{\lambda}: E_{g(\lambda)}\left(\Delta_{G}\right) \longrightarrow E_{\lambda}\left(\Delta_{X_{0}}\right) \\
\left(U_{\lambda} a\right)_{e}(x):=\frac{\sqrt{\ell}}{\sqrt{2} \sin (\ell \sqrt{\lambda})}\left(a\left(\partial_{-} e\right) \sin ((\ell-x) \sqrt{\lambda})+a\left(\partial_{+} e\right) \sin (x \sqrt{\lambda})\right)
\end{gathered}
$$

is an isometry from the eigenspace of $\Delta_{G}$ w.r.t. the eigenvalue $g(\lambda)$ onto the eigenspace of $\Delta_{X_{0}}$ w.r.t. the eigenvalue $\lambda \notin \Sigma^{\mathrm{D}}$. In particular, multiplicities of eigenvalues are preserved. Furthermore, $\lambda$ is isolated in $\sigma\left(\Delta_{X_{0}}\right)$ iff $g(\lambda)$ is isolated

\footnotetext{
${ }^{3}$ Note that $\sigma_{\mathrm{c}}$ does in general not coincide with the continuous spectrum, cf. [RS80.
} 
in $\sigma\left(\Delta_{G}\right)$, so the statement on the discrete spectrum follows. For the essential spectrum note that we already have the statement for the entire spectrum and for the discrete spectrum, and that $\sigma_{\text {ess }}=\sigma \backslash \sigma_{\text {disc }}$.

Since the spectrum of the discrete Laplacian has been explored in many cases, the previous theorem allows us to determine the corresponding spectrum of the equilateral metric graph leading to interesting examples of graph-like manifolds. For simplicity, we fix the edge length to $\ell=1$.

Homogeneous trees. Let $G$ be a homogeneous routed tree of degree $d_{0} \geq 3$, then

$$
\sigma_{\mathrm{p}}\left(\Delta_{G}\right)=\emptyset \quad \text { and } \quad \sigma_{\mathrm{c}}\left(\Delta_{G}\right)=\left[1-\frac{2 \sqrt{d_{0}-1}}{d_{0}}, 1+\frac{2 \sqrt{d_{0}-1}}{d_{0}}\right] .
$$

Theorem 3.6] now describes $\sigma_{\mathrm{c}}\left(\Delta_{X_{0}}\right)$. Since $\sigma\left(\Delta_{G}\right)=\sigma_{\mathrm{c}}\left(\Delta_{G}\right) \subsetneq[0,2]$, the metric graph Laplacian has spectral gaps (i.e., no spectrum at) $I_{0}:=\left(0, \omega_{0}^{2}\right)$ and

$$
I_{k}:=\left(\left(k \pi-\omega_{0}\right)^{2},(k \pi)^{2}\right) \cup\left((k \pi)^{2},\left(k \pi+\omega_{0}\right)^{2}\right), \quad k \in \mathbb{N},
$$

where $\omega_{0}=\arccos \left(2 \sqrt{d_{0}-1} / d_{0}\right)$. A more detailed analysis done in Ca97 shows that $\sigma_{\mathrm{p}}\left(\Delta_{X_{0}}\right)=\Sigma^{\mathrm{D}}$. All eigenvalues have infinite multiplicity, so $\sigma\left(\Delta_{X_{0}}\right)$ is purely essential and has band-gap structure with gaps exactly at $I_{k}, k \in \mathbb{N}_{0}$. In particular, inf $\sigma\left(\Delta_{X_{0}}\right)=\omega_{0}^{2}>0$ and we have:

Theorem 3.7. Suppose $X_{\varepsilon}$ is a family of uniform graph-like manifolds associated to the regular tree of degree $d_{0} \geq 3$ with equal edge lengths. Then the Laplacian on $X_{\varepsilon}$ has spectral gaps and its essential spectrum is non-empty near any $\lambda \in \sigma_{\mathrm{ess}}\left(\Delta_{X_{0}}\right)$ provided $\varepsilon$ is small enough (cf. Corollary A.14). Furthermore, $\Sigma_{0}\left(X_{\varepsilon}\right):=\inf \sigma\left(\Delta_{X_{\varepsilon}}\right) \rightarrow \omega_{0}^{2}$ as $\varepsilon \rightarrow 0$ and in particular, $\Sigma_{0}\left(X_{\varepsilon}\right) \geq c>0$ for $\varepsilon$ small enough.

Remark 3.8. Note that if $X_{\varepsilon}$ would be a Riemannian covering of a compact manifold $M_{\varepsilon} \cong X_{\varepsilon} / \Gamma$, then the non-amenability of $\Gamma$ (if e.g. $\Gamma$ contains the non-abelian free group $\mathbb{Z}^{* 2}$ as subgroup) implies $\Sigma_{0}\left(X_{\varepsilon}\right)>0$ (cf. Br81a]). An unrooted tree of degree $d_{0}$ can be considered as unoriented Cayley graph of the group $\mathbb{Z}_{2}^{* d_{0}}$ (free product of $d_{0}$ groups of order 2 ) with respect to the generators $\gamma_{i}, i=1, \ldots, d_{0}$. But the elements of order 2 act on a corresponding graph-like manifold as reflections. In particular, these elements have fixed points and the quotient is no longer a smooth manifold so we cannot use the covering argument here in order to show $\Sigma_{0}\left(X_{\varepsilon}\right)>0$.

Remark 3.9. There is a nice upper estimate for $\Sigma_{\text {ess }}(X):=\inf \sigma_{\text {ess }}\left(\Delta_{X}\right)$ : Denote by

$$
\mu(X):=\lim _{r \rightarrow \infty} \frac{1}{r} \log \operatorname{vol}_{d} B\left(x_{0}, r\right)
$$

the growth rate of a ball of radius $r$ in $X$. It can be easily seen that $\mu(X)$ is independent of $x_{0}$. Brooks $\left.\mathrm{Br} 81 \mathrm{~b}\right]$ showed that if $\operatorname{vol}_{d}(X)=\infty$ then

$$
\Sigma_{\text {ess }}(X) \leq \frac{1}{4} \mu(X)^{2}
$$

A priori, this estimate is not sharp as there are amenable groups $\pi$ of exponential growth (cf. M68]): For the universal cover $\widetilde{M}$ of a compact manifold $M$ with amenable fundamental group $\pi_{1}(M)=\pi$ we have $\Sigma_{\text {ess }}(\widetilde{M})=0$ but $\mu(\widetilde{M})>0$. 
In our case, we can easily calculate $\mu\left(X_{\varepsilon}\right)$ approximately: If $x_{0}$ is a point in the root vertex neighbourhood then an increase of $r$ by $\ell_{0}=1$ encounters another generation in the tree. Therefore $\operatorname{vol}_{d} B\left(x_{0}, r\right) \approx \operatorname{vol}_{d} \hat{U}_{\varepsilon, v}\left(\left(d_{0}-1\right)^{r}-1\right)$ for large $r$ where $\hat{U}_{\varepsilon, v}$ is a (sample) vertex neighbourhood together with the $d_{0}$ adjacent half edge neighbourhoods. We obtain $\mu\left(X_{\varepsilon}\right) \approx \log \left(d_{0}-1\right)$ and in particular, our estimate shows

$$
\Sigma_{0}\left(X_{\varepsilon}\right)=\Sigma_{\text {ess }}\left(X_{\varepsilon}\right) \approx \arccos ^{2}\left(\frac{2 \sqrt{d_{0}-1}}{d_{0}}\right)<\frac{1}{4} \log ^{2}\left(d_{0}-1\right) \approx \frac{1}{4} \mu\left(X_{\varepsilon}\right)^{2}
$$

if $d_{0} \geq 3$. The inequality provides an (approximative) lower bound on $\mu\left(X_{\varepsilon}\right)$ and the difference of the LHS and RHS is e.g. smaller than $1 \%$ if $d_{0}=3$ ). For further estimates of this type using isoperimetric constants we refer to $\mathrm{Br} 81 \mathrm{~b}$. We only want to mention here that isoperimetric constants on graph-like manifolds can quite easily be calculated.

Sierpiński graphs. Let us give another interesting example admitting a fractal spectrum. Let $G_{1}$ be the complete graph with three vertices. Suppose $G_{n}=$ $\left(V_{n}, E_{n}\right)$ has already been constructed. Then $G_{n+1}$ is obtained from three disjoint copies $G_{n}^{(i)}(i=1,2,3)$ of $G_{n}$ and the equivalent relation $\sim$, i.e., $G_{n+1}:=G_{n}^{(1)} \uplus$ $G_{n}^{(2)} \uplus G_{n}^{(3)} / \sim$ where $\sim$ identifies the vertex $v_{i}\left(G_{n}^{(j)}\right)$ with $v_{j}\left(G_{n}^{(i)}\right), i \neq j, i, j=$ $1,2,3$. Here, $v_{1}\left(G_{n}\right)$ denotes the lower left vertex of degree $2, v_{2}\left(G_{n}\right)$ the lower right vertex of degree 2 and $v_{3}\left(G_{n}\right)$ the upper vertex of degree 2 (cf. Figure 4). Note that each $G_{n}$ has exactly three vertices of degree 2, the other have degree 4. Furthermore, $\left|V_{n}\right|=\left(3^{n}+1\right) / 2$ and $\left|E_{n}\right|=3^{n}$. Now, $G_{n}$ embeds into $G_{n+1}$ via $G_{n}=G_{n}^{(1)} \hookrightarrow G_{n+1}$. Finally, the Sierpińksi graph is given by $G:=\bigcup_{n \in \mathbb{N}} G_{n}$ (cf. Figure 4). Note that $G$ has one vertex of degree 2, all other vertices have degree 4 . The nature of the spectrum of the discrete Laplacian $\Delta_{G}$ was calculated by [T98, Thm. 2]: We have

$$
\sigma\left(\Delta_{G}\right)=J \cup D, \quad D:=\left\{\frac{3}{2}\right\} \cup \bigcup_{n=0}^{\infty} p^{-n}\left\{\frac{3}{4}\right\}, \quad J:=\bar{D},
$$

where $p(z):=z(5-4 z)$. and $p^{-n}$ is the $n$-th pre-image, i.e., $p^{-n}\{3 / 4\}=\{z \in$ $\left.\mathbb{R} \mid p^{\circ n}(z)=3 / 4\right\}$. The spectrum of $\Delta_{G}$ is pure point, each eigenvalue has multiplicity $\infty$, so the spectrum is also purely essential. The set $D$ consists of the isolated eigenvalues and the set $J$ is a Cantor set of Lebesgue measure 0 (the Julia set of the polynomial $p$ ). Due to Theorem 3.6 the spectrum of the metric graph Laplacian $\Delta_{X_{0}}$ (with $\left.\ell=1\right)$ is pure point and purely essential. It is given by

$$
\sigma\left(\Delta_{X_{0}}\right) \backslash \Sigma^{\mathrm{D}}=g^{-1}(D) \cup g^{-1}(J \backslash\{0\}) .
$$

Since $g(\lambda)=1-\cos \sqrt{\lambda}$ is a local homeomorphism mapping measure 0 set into measure 0 sets and vice versa, $g^{-1}(D)$ consists of isolated eigenvalues of $\Delta_{X_{0}}$ of infinite multiplicity and $g^{-1}(J \backslash\{0\})$ is a Cantor set of measure 0 (away from $\left.\Sigma^{\mathrm{D}}\right)$.

Now our spectral convergence result on graph-like manifolds leads to an example of a family of smooth manifolds approaching a fractal spectrum:

Theorem 3.10. Suppose $X_{\varepsilon}$ is a family of uniform graph-like manifolds associated to the Sierpinski graph with equal edge lengths. Then the essential spectrum 


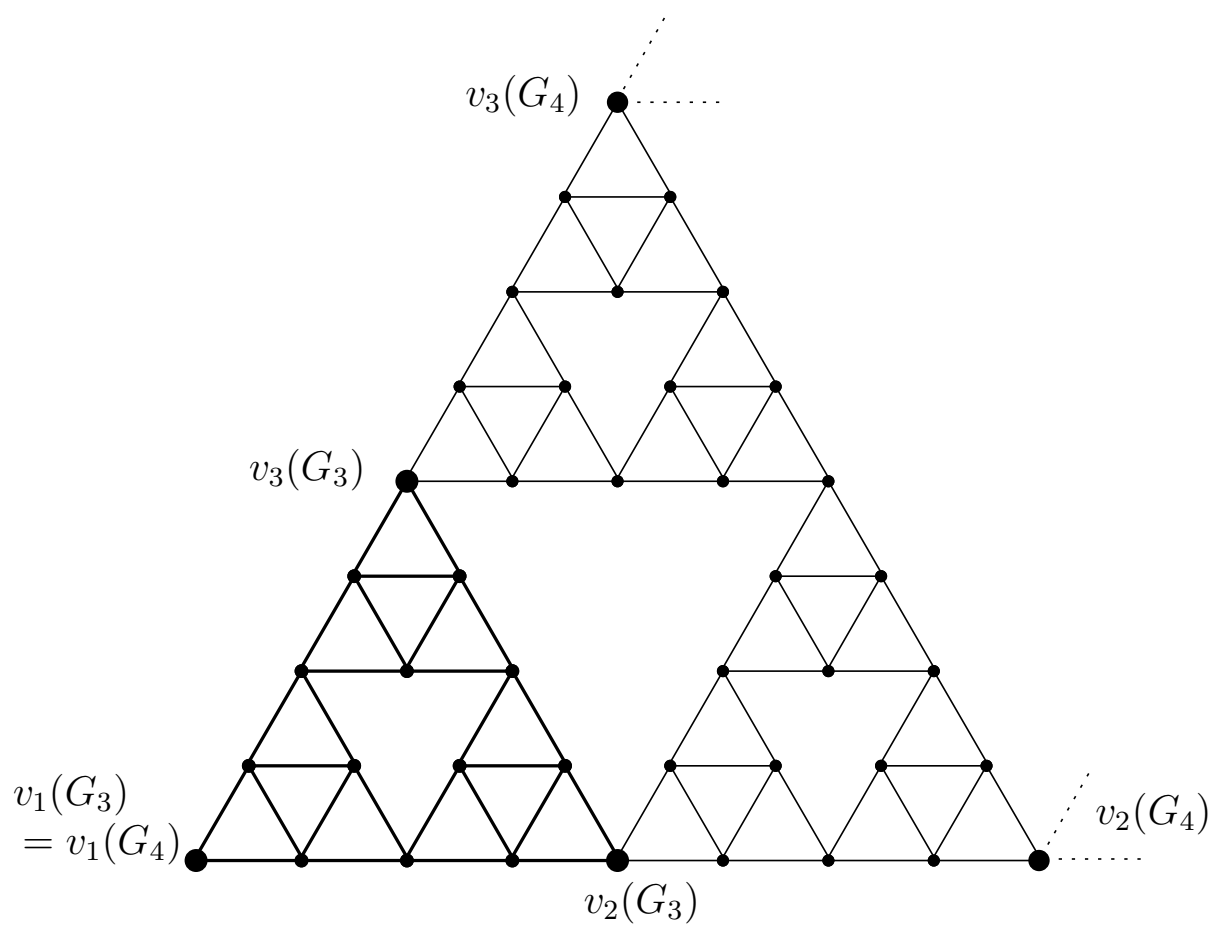

Figure 4. The first four generations $G_{4}$ of the infinite Sierpiński graph, each edge having unit length. The graph $G_{3}$ is denoted with thick edges and is naturally embedded into $G_{4}$.

of the Laplacian on $X_{\varepsilon}$ approaches the fractal spectrum of $\Delta_{X_{0}}$ in any fixed interval $[0, \Lambda]$. The discrete spectrum of $\Delta_{X_{s}}$ is either empty or merges into the essential spectrum as $\varepsilon \rightarrow 0$ (cf. Corollary A.14). In particular, $\sigma\left(\Delta_{X_{\varepsilon}}\right)$ has an arbitrary large number of spectral gaps in the compact interval $[0, \Lambda]$ provided $\varepsilon$ is small enough.

3.4. The decoupling case. We obtain similar convergence results on noncompact graph-like manifolds $X_{\varepsilon}$ with slower decay of vol $U_{\varepsilon, v}$ (i.e., large vertex neighbourhoods $U_{\varepsilon, v}$ ) as discussed in the compact case in KuZ03 and EP05, Sec.5-8]. If for example the vertex volume vol $U_{\varepsilon, v}$ decays slower than the edge volume $\operatorname{vol} U_{\varepsilon, e}$ then the Laplacian on $X_{\varepsilon}$ converges also in the non-compact case to the decoupled limit operator $H:=\bigoplus_{e} H_{e} \oplus 0$ acting in the enlarged Hilbert space $\mathcal{H}:=\mathrm{L}_{2}(X)_{0} \oplus \mathbb{C}^{V}$. Here, $H_{e}$ is the Dirichlet operator on the interval $\left(0, \ell_{e}\right) \cong e$ and 0 is the null operator on $\mathbb{C}^{V}$. Therefore, the limit operator has pure point spectrum

$$
\sigma(H)=\left\{\lambda_{k}(e):=\frac{\pi^{2} k^{2}}{\ell_{e}^{2}} \mid e \in E, k \in \mathbb{N}_{0}\right\}
$$

and the multiplicity of $\lambda$ is $|V|$ if $\lambda=0$ and $\left|\left\{e \in E \mid \lambda=\lambda_{k}(e)\right\}\right|$ if $\lambda>0$. We omit the details here since the proof is rather similar to the non-decoupling case treated below. Note that we need here some uniformity assumptions on the vertex neighbourhood $U_{\varepsilon, v}$ ensuring that $U_{\varepsilon, v}$ does not become too small, e.g., we need a lower bound on $\operatorname{vol} U_{\varepsilon, v}^{-}$. Here $U_{\varepsilon, v}^{-}$is the subset of $U_{\varepsilon, v}$ where the metric satisfies $g_{\varepsilon, v} \cong \varepsilon^{2 \alpha} g_{v}$ and $0<\alpha<(d-1) / d$ (cf. Remark 2.73] and 2.74). 
Theorem 3.11. Suppose that $X_{\varepsilon}$ is a graph-like manifold with large vertex neighbourhoods associated to a metric graph $X_{0}$ with edge length $\left(\ell_{e}\right)_{e}$. Then the Laplacian on $X_{\varepsilon}$ (with Neumann boundary conditions, if $\partial X_{\varepsilon} \neq \emptyset$ ) approaches the decoupled operator $H$. In particular, its spectrum converges to the set $\sigma(H)$ given in (3.6) in any compact spectral interval.

In a similar way, the case of Dirichlet boundary conditions and small junctions as in [P05] can be extended to the non-compact case. The Laplacian on $X_{\varepsilon}$ with Dirichlet boundary conditions has to be shifted by $\lambda_{1}^{\mathrm{D}}(F) / \varepsilon^{2}$ where $\lambda_{1}^{\mathrm{D}}(F)$ is the first Dirichlet eigenvalue of the cross section. The limit operator also decouples and consists of the Dirichlet eigenvalues only.

Since the graph structure is no more visible in the limit due to the decoupling, we just give a simple example of a graph consisting of a half-line with one vertex of degree 1 at 0 and the others of degree 2 . We label the edges by $n \in \mathbb{N}$. By an appropriate choice of the length $\ell_{e}$ we can construct a manifold with certain spectral properties. If we consider e.g. $\ell_{n}:=\sqrt{n} / \pi$ then $\lambda_{k}(n)=k^{2} / n$. Since every rational number $r$ can be written in the form $r=k^{2} / n(r=p / q=$ $\left.p^{2} /(p q)\right)$, the operator $H$ has dense point spectrum consisting of all non-negative rational numbers. Therefore the spectrum of $H$ is $[0, \infty)$ and purely essential. Our analysis here is too weak to say anything more on the nature of the Laplacian on the graph-like manifold $X_{\varepsilon}$ with large vertex neighbourhoods than it is purely essential in any bounded spectral interval. We expect that $\Delta_{X_{\varepsilon}}$ also has pure point spectrum, but one needs arguments from scattering theory to prove this.

Other choices of the length are possible, e.g., a set of rationally independent length $\ell_{e}, e \in E$. Once one is able to determine $\sigma(H)$ by number theoretical arguments one has a Laplacian $\Delta_{X_{\varepsilon}}$ with a spectrum close to this set.

\section{A. Appendix}

In the appendix we prove our main technical tool, the convergence results for arbitrary pairs of self-adjoint non-negative operators and Hilbert spaces $(H, \mathcal{H})$ and $(\widetilde{H}, \widetilde{\mathcal{H}})$ being close to each other (cf. Definition A.1). Although most of the techniques are standard, we repeat the arguments here since usually, one has a fixed Hilbert space and a careful analysis of the dependence on some parameter entering in the operator and Hilbert space is not necessary. We do not mention the parameter explicitely but express all convergence results in terms of a "distance" $\delta$ of $(H, \mathcal{H})$ and $(\widetilde{H}, \widetilde{\mathcal{H}})$.

A.1. Scale of Hilbert spaces associated with a non-negative operator. To a Hilbert space $\mathcal{H}$ with inner product $\langle\cdot, \cdot\rangle$ and norm $\|\cdot\|$ together with a non-negative, unbounded, operator $H$, we associate the scale of Hilbert spaces

$$
\mathcal{H}_{k}:=\operatorname{dom}(H+1)^{k / 2}, \quad\|u\|_{k}:=\left\|(H+1)^{k / 2} u\right\|, \quad k \geq 0 .
$$

For negative exponents, we define

$$
\mathcal{H}_{-k}:=\mathcal{H}_{k}^{*}
$$

Note that $\mathcal{H}=\mathcal{H}_{0}$ embeds naturally into $\mathcal{H}_{-k}$ via $u \mapsto\langle u, \cdot\rangle$ since

$$
\|\langle u, \cdot\rangle\|_{-k}=\sup _{v \in \mathcal{H}_{k}} \frac{|\langle u, v\rangle|}{\|v\|_{k}}=\sup _{w \in \mathcal{H}_{0}} \frac{\left|\left\langle R^{k / 2} u, w\right\rangle\right|}{\|w\|_{0}}=\left\|R^{k / 2} u\right\|_{0},
$$


where

$$
R:=(H+1)^{-1}
$$

denotes the resolvent of $H \geq 0$. The last equality used the natural identification $\mathcal{H} \cong \mathcal{H}^{*}$ via $u \mapsto\langle u, \cdot\rangle$. Therefore, we can interprete $\mathcal{H}_{-k}$ as the completion of $\mathcal{H}$ in the norm $\|\cdot\|_{-k}$. With this identification, we have

$$
\|u\|_{-k}=\sup _{v \in \mathcal{H}_{k}} \frac{|\langle u, v\rangle|}{\|v\|_{k}}, \quad \text { for all } k \in \mathbb{R} .
$$

For a second Hilbert space $\widetilde{\mathcal{H}}$ with inner product $\langle\cdot, \cdot\rangle$ and norm $\|\cdot\|$ together with a non-negative, unbounded, operator $\widetilde{H}$, we define in the same way a scale of Hilbert spaces $\widetilde{\mathcal{H}}_{k}$ with norms $\|\cdot\|_{k}$.

Guided by the classical application $H=\Delta_{X}$ in $\mathcal{H}=\mathrm{L}_{2}(X)$ for a complete manifold $X$, we call $k$ the regularity order. In this case, $\mathcal{H}_{k}$ corresponds to the $k$-th Sobolev space $\mathrm{H}^{k}(X)$.

A.2. Operators on scales. Suppose we have two scales of Hilbert spaces $\mathcal{H}_{k}$, $\widetilde{\mathcal{H}}_{k}$ associated to the non-negative operators $H, \widetilde{H}$ with resolvents $R:=(H+1)^{-1}$, $\widetilde{R}:=(\widetilde{H}+1)^{-1}$, respectively. The norm of an operator $A: \mathcal{H}_{k} \longrightarrow \widetilde{\mathcal{H}}_{-\widetilde{k}}$ is

$$
\|A\|_{k \rightarrow-\widetilde{k}}:=\sup _{u \in \mathcal{H}_{k}} \frac{\|A u\|_{-\widetilde{k}}}{\|u\|_{k}}=\left\|\widetilde{R}^{\widetilde{k} / 2} A R^{k / 2}\right\|_{0 \rightarrow 0} .
$$

The norm of the adjoint $A^{*}: \widetilde{\mathcal{H}}_{\widetilde{k}} \longrightarrow \mathcal{H}_{-k}$ is given by

$$
\left\|A^{*}\right\|_{\widetilde{k} \rightarrow-k}=\|A\|_{k \rightarrow-\widetilde{k}} \text {. }
$$

Furthermore,

$$
\|A\|_{k \rightarrow-\widetilde{k}} \leq\|A\|_{m \rightarrow-\widetilde{m}} \quad \text { provided } \quad k \geq m, \widetilde{k} \geq \widetilde{m}
$$

since

$$
\|A\|_{k \rightarrow-\widetilde{k}}=\left\|\widetilde{R}^{\widetilde{k} / 2} A R^{k / 2}\right\|_{0 \rightarrow 0}=\left\|\widetilde{R}^{(\widetilde{k}-\widetilde{m}) / 2} \widetilde{R}^{\widetilde{m} / 2} A R^{m / 2} R^{(k-m) / 2}\right\|_{0 \rightarrow 0} \leq\|A\|_{m \rightarrow-\widetilde{m}}
$$

and $\|R\|,\|\widetilde{R}\| \leq 1$.

A.3. Closeness assumption. In this section we state our main assumptions on the two operators $H$ and $\widetilde{H}$ acting in the Hilbert spaces $\mathcal{H}$ and $\widetilde{\mathcal{H}}$. We think of $(\widetilde{H}, \widetilde{\mathcal{H}})$ being a perturbation of $(H, \mathcal{H})$, or that $(H, \mathcal{H})$ describes a simplified model (say, on a metric graph $X_{0}$ ) which is close to a more complicated model given by $(\widetilde{H}, \widetilde{\mathcal{H}})$ (say, on a graph-like manifold $X_{\varepsilon}$ ). We want to state assumptions under which $(H, \mathcal{H})$ and $(\widetilde{H}, \widetilde{\mathcal{H}})$ are close in a sense to be specified in Definition A.1.

Let us explain the following concept of quasi-unitary operators in the case of unitary operators (cf. also Example A.2): Suppose we have a unitary operator $J: \mathcal{H} \longrightarrow \widetilde{\mathcal{H}}$ with inverse $J^{\prime}=J^{*}: \widetilde{\mathcal{H}} \longrightarrow \mathcal{H}$ respecting the quadratic form domains, i.e. $J_{1}:=J \uparrow_{\mathcal{H}_{1}}: \mathcal{H}_{1} \longrightarrow \widetilde{\mathcal{H}}_{1}$ and $J_{1}^{\prime}=J^{*} \uparrow_{\widetilde{\mathcal{H}}_{1}}: \widetilde{\mathcal{H}}_{1} \longrightarrow \mathcal{H}_{1}$. If

$$
J_{1}^{\prime *} H=\widetilde{H} J_{1}
$$

then $H$ and $\widetilde{H}$ are unitarily equivalent and have therefore the same spectral properties. The main point here is that $J$ respects the quadratic form domain 
and therefore, $J_{1}^{\prime *}: \mathcal{H}_{-1} \longrightarrow \widetilde{\mathcal{H}}_{-1}$ is an extension of $J: \mathcal{H} \longrightarrow \widetilde{\mathcal{H}}$. In this sense, the above equality says that $J$ is an intertwining operator.

We want to lessen the assumption such that the spectral properties are not the same but still at close quarters. We now start with the definition of $\delta$-closeness:

Definition A.1. Suppose we have linear operators

$$
\begin{array}{rlrl}
J: \mathcal{H} & \longrightarrow \widetilde{\mathcal{H}}, & J^{\prime}: \widetilde{\mathcal{H}} & \longrightarrow \mathcal{H} \\
J_{1}: \mathcal{H}_{1} & \longrightarrow \widetilde{\mathcal{H}}_{1}, & J_{1}^{\prime}: \widetilde{\mathcal{H}}_{1} \longrightarrow \mathcal{H}_{1} .
\end{array}
$$

Let $\delta>0$ and $k \geq 1$. We say that $(H, \mathcal{H})$ and $(\widetilde{H}, \widetilde{\mathcal{H}})$ are $\delta$-close with respect to the quasi-unitary maps $\left(J, J_{1}\right)$ and $\left(J^{\prime}, J_{1}^{\prime}\right)$ of order $k$ iff the following conditions are fulfilled:

$$
\begin{aligned}
\left\|J-J_{1}\right\|_{1 \rightarrow 0} & \leq \delta, & \left\|J^{\prime}-J_{1}^{\prime}\right\|_{1 \rightarrow 0} \leq \delta \\
\left\|J-J^{\prime *}\right\|_{0 \rightarrow 0} & \leq \delta, & \\
\left\|\widetilde{H} J_{1}-J_{1}^{\prime *} H\right\|_{k \rightarrow-1} & \leq \delta, & \\
\left\|\mathbb{1}-J^{\prime} J\right\|_{1 \rightarrow 0} & \leq \delta, & \left\|\mathbb{1}-J J^{\prime}\right\|_{1 \rightarrow 0} \leq \delta \\
\|J\|_{0 \rightarrow 0} & \leq 2, & \left\|J^{\prime}\right\|_{0 \rightarrow 0} \leq 2 .
\end{aligned}
$$

Note that all operators make sense on the given domains, e.g.,

$$
\left(\widetilde{H} J_{1}-J_{1}^{\prime *} H\right)\left\lceil_{\mathcal{H}_{k}}=\left(\widetilde{H} J_{1}-J_{1}^{\prime *} H\right) \mathbb{1}_{k \rightarrow 1}: \mathcal{H}_{k} \longrightarrow \widetilde{\mathcal{H}}_{-1}\right.
$$

where $\mathbb{1}_{k \rightarrow 1}: \mathcal{H}_{k} \longrightarrow \mathcal{H}_{1}$ is the natural inclusion map. Strictly speaking, we should also write $J \mathbb{1}_{1 \rightarrow 0}-\widetilde{\mathbb{1}}_{1 \rightarrow 0} J_{1}$ in (A.9) and $\left(\mathbb{1}_{0 \rightarrow 0}-J^{\prime} J\right) \mathbb{1}_{1 \rightarrow 0}$ in (A.12), e.g., but we refrain from it in order to keep the notation readable.

We can interprete A.11 in the sense that $J_{1}$ and $J_{1}^{\prime}$ are quasi-intertwining operators. Since (A.9) assures the closeness of $J$ and $J_{1}$ resp. $J^{\prime}$ and $J_{1}^{\prime}$ we call $J$ resp. $J^{\prime}$ also quasi-intertwining. Furthermore, (A.12) together with (A.10) says that $J$ and $J^{\prime}$ are quasi-unitary. In our application of a graph-like manifold converging to a metric graph, cf. Section 2), the regularity order $k$ equals 1 . In this case, the assumptions are symmetric in $H$ and $\widetilde{H}$, but we will also meet situations in a forthcoming paper where $k>1$ is needed.

For concrete applications, the following equivalent characterisation of (A.9)A.13) will be useful:

$$
\begin{array}{rlrl}
\left\|J f-J_{1} f\right\|_{0} & \leq \delta\|f\|_{1}, & \left\|J^{\prime} u-J_{1}^{\prime} u\right\|_{0} & \leq \delta\|u\|_{1} \\
\left|\langle J f, u\rangle-\left\langle f, J^{\prime} u\right\rangle\right| & \leq \delta\|f\|_{0}\|u\|_{0} & & \text { A. } \\
\mid \widetilde{h}\left(J_{1} f, 10\right) & \\
\left\|f-J^{\prime} J f\right\|_{0} & \leq \delta\|f\|_{1}, & \left\|u-J J^{\prime} u\right\|_{0} & \leq \delta\|u\|_{1} \\
\|J f\|_{0} & \leq 2\|f\|_{0}, & \left\|J^{\prime} u\right\|_{0} & \leq 2\|u\|_{0}
\end{array}
$$

for all $f, u$ in the appropriate spaces. Here, $\mathfrak{h}$ and $\widetilde{\mathfrak{h}}$ denote the sesquilinear forms associated to $H$ and $\widetilde{H}$, i.e., $\mathfrak{h}(f, g)=\left\langle H^{1 / 2} f, H^{1 / 2} g\right\rangle$ for $f, g \in \mathcal{H}_{1}$ and similarly for $\widetilde{\mathfrak{h}}$.

Let us illustrate the above abstract setting in the following example of norm resolvent convergence in a fixed Hilbert space: 
Example A.2. Suppose that $\widetilde{\mathcal{H}}=\mathcal{H}, J=J^{\prime}=\mathbb{1}, J_{1}=J_{1}^{\prime}=\mathbb{1}, k=1$ and $\delta=\delta_{n} \rightarrow 0$ as $n \rightarrow \infty$. Assume in addition that the quadratic form domains of $H$ and $\widetilde{H}=H_{n}$ agree. Now the only non-trivial assumption in Definition A.1 is Equation (A.11), which is equivalent to

$$
\left\|H_{n}-H\right\|_{1 \rightarrow-1}=\left\|R_{n}^{1 / 2}\left(H_{n}-H\right) R^{1 / 2}\right\|_{0 \rightarrow 0} \rightarrow 0
$$

whereas $H_{n} \rightarrow H$ in norm resolvent convergence means

$$
\left\|R_{n}-R\right\|_{0 \rightarrow 0}=\left\|R_{n}\left(H_{n}-H\right) R\right\|_{0 \rightarrow 0}=\left\|H_{n}-H\right\|_{2 \rightarrow-2} \rightarrow 0
$$

as $n \rightarrow \infty$. Therefore, we see that our assumption (A.11) implies the norm resolvent convergence but not vice versa.

Remark A.3. We have expressed the closeness of certain quantities in dependence on the initial closeness data $\delta>0$. Although, in our applications, $(\widetilde{H}, \widetilde{\mathcal{H}})$ will depend on some parameter $\varepsilon>0$ with $\delta=\delta(\varepsilon) \rightarrow 0$ as $\varepsilon \rightarrow 0$ we prefer to express the dependence only in terms of $\delta$. In particular, an assertion like $\|J R-\widetilde{R} J\| \leq 4 \delta$ means that it is true for all $(H, \mathcal{H})$ and $(\widetilde{H}, \widetilde{\mathcal{H}})$ being $\delta$-close with respect to $\left(J, J_{1}\right)$ and $\left(J^{\prime}, J_{1}^{\prime}\right)$. In this sense, $(H, \mathcal{H})$ and $(\widetilde{H}, \widetilde{\mathcal{H}})$ should be considered as "variables" being close to each other.

We deduce the following simple estimates:

Lemma A.4. Suppose that Assumption (A.10), (A.12) and (A.13) are fulfilled, then

$$
\|f\|_{0}-\delta^{\prime}\|f\|_{1} \leq\|J f\|_{0} \leq\|f\|_{0}+\delta^{\prime}\|f\|_{1} \quad \text { with } \quad \delta^{\prime}:=\sqrt{3 \delta}
$$

and similarly for $J^{\prime}$.

Proof. We calculate

$$
\begin{aligned}
&\left|\|J f\|^{2}-\|f\|^{2}\right|=\left|\left\langle\left(J^{*} J-\mathbb{1}\right) f, f\right\rangle\right| \leq\left|\left\langle\left(J^{*}-J^{\prime}\right) J f, f\right\rangle\right|+\left|\left\langle\left(J^{\prime} J-\mathbb{1}\right) f, f\right\rangle\right| \\
& \leq\left\|J^{*}-J^{\prime}\right\|_{0 \rightarrow 0}\|J f\|_{0}\|f\|_{0}+\left\|J^{\prime} J-\mathbb{1}\right\|_{1 \rightarrow 0}\|f\|_{1}\|f\|_{0} \leq 3 \delta\|f\|_{1}^{2}
\end{aligned}
$$

and the result follows.

A.4. Resolvent convergence and functional calculus. In this section we prove our result on resolvent convergence. More precisely, we estimate the errors in terms of $\delta$. All the results below are valid for pairs of non-negative operators and Hilbert spaces $(H, \mathcal{H})$ and $(\widetilde{H}, \widetilde{\mathcal{H}})$ which are $\delta$-close of order $k$. We set

$$
m:=\max \{0, k-2\}
$$

as regularity order for the resolvent difference. Note that $m=0$ if $k=1$ (as in our application) or $k=2$.

Theorem A.5. Suppose (A.9), (A.10) and (A.11), then

$$
\begin{gathered}
\|\widetilde{R} J-J R\|_{m \rightarrow 0}=\|J H-\widetilde{H} J\|_{2+m \rightarrow-2} \leq 4 \delta, \\
\left\|\widetilde{R}^{j} J-J R^{j}\right\|_{m \rightarrow 0} \leq 4 j \delta
\end{gathered}
$$

for all $j \in \mathbb{N}$. 
Proof. We start with the equation

$$
J H-\widetilde{H} J=\left(J-J^{\prime *}\right) H+\left(J^{\prime}-J_{1}^{\prime}\right)^{*} H+\left(J_{1}^{*} H-\widetilde{H} J_{1}\right)+\widetilde{H}\left(J_{1}-J\right)
$$

considered as bounded operator from $\mathcal{H}_{2+m}$ to $\widetilde{\mathcal{H}}_{-2}$. Using (A.6) and (A.7) yields

$$
\begin{aligned}
& \|\widetilde{R} J-J R\|_{m \rightarrow 0}=\|\widetilde{R}(J H-\widetilde{H} J) R\|_{m \rightarrow 0}=\|J H-\widetilde{H} J\|_{2+m \rightarrow-2} \\
& \leq\left\|J-J^{\prime *}\right\|_{m \rightarrow-2}+\left\|J^{\prime}-J_{1}^{\prime}\right\|_{2 \rightarrow-m}+\left\|J_{1}^{* *} H-\widetilde{H} J_{1}\right\|_{2+m \rightarrow-2}+\left\|J_{1}-J\right\|_{2+m \rightarrow 0} \\
& \leq\left\|J-J^{\prime *}\right\|_{0 \rightarrow 0}+\left\|J^{\prime}-J_{1}^{\prime}\right\|_{1 \rightarrow 0}+\left\|J_{1}^{\prime *} H-\widetilde{H} J_{1}\right\|_{k \rightarrow-1}+\left\|J_{1}-J\right\|_{1 \rightarrow 0} \leq 4 \delta,
\end{aligned}
$$

i.e., the assertion (A.16). For the second estimate we use the resolvent identity

$$
\widetilde{R}^{j} J-J R^{j}=\sum_{i=0}^{j-1} \widetilde{R}^{j-1-i}(\widetilde{R} J-J R) R^{i}
$$

and conclude

$$
\left\|\widetilde{R}^{j} J-J R^{j}\right\|_{m \rightarrow 0} \leq \sum_{i=0}^{j-1}\left\|\widetilde{R}^{j-1-i}\right\|_{0 \rightarrow 0}\|\widetilde{R} J-J R\|_{m \rightarrow 0}\left\|R^{i}\right\|_{m \rightarrow m} \leq 4 j \delta
$$

using the estimate for $j=1$. Note that $\|R\|_{m \rightarrow m} \leq 1$ for any $m$ and similarly for $\widetilde{R}$.

Remark A.6. Observe that we cannot obtain a better result using the quasiunitary operator $J$ although we loose regularity order at some stages. The best what we can expect (in the case $k=1$ ) is the estimate

$$
\left\|\widetilde{R} J_{1}^{\prime *}-J_{1} R\right\|_{-1 \rightarrow 1} \leq 4 \delta
$$

which follows from

$$
\begin{aligned}
& (\widetilde{H}+1)^{1 / 2}\left(\widetilde{R} J_{1}^{\prime *}-J_{1} R\right)(H+1)^{1 / 2} \\
& \quad=\widetilde{R}^{1 / 2}\left[\left(J_{1}^{\prime *} H-\widetilde{H} J_{1}\right)+\left(J_{1}^{\prime *}-J^{* *}\right)+\left(J^{\prime *}-J\right)+\left(J-J_{1}\right)\right] R^{1 / 2}
\end{aligned}
$$

and the assumptions.

On the other hand, if we assume that $\|J H-\widetilde{H} J\|_{2+m \rightarrow-2} \leq \widetilde{\delta}$, i.e.,

$$
|\langle J H f, u\rangle-\langle J f, \widetilde{H} u\rangle| \leq \widetilde{\delta}\|f\|_{2+m}\|u\|_{2}
$$

for all $f \in \mathcal{H}_{2+m}, u \in \widetilde{\mathcal{H}}_{2}$ then we directly obtain the resolvent estimate (A.16) with $\widetilde{\delta}=4 \delta$. Although Assumption (A.19) is weaker than A.9)-A.11) (cf. Example A.2) it is often easier in our applications to deal with the quadratic form domains, even if one needs the additional operators $J^{\prime}, J_{1}$ and $J_{1}^{\prime}$ and the stronger estimates (A.9)- A.11).

We want to extend our results to more general functions $\varphi(H)$ of the operator $H$ and similarly for $\widetilde{H}$. We start with continuous functions on $\mathbb{R}_{+}:=[0, \infty)$ such that $\lim _{\lambda \rightarrow \infty} \varphi(\lambda)$ exist, i.e., with functions continuous on $\overline{\mathbb{R}}_{+}:=[0, \infty]$. We denote this space by $\mathrm{C}\left(\overline{\mathbb{R}}_{+}\right)$.

Theorem A.7. Suppose that (A.9), (A.10), (A.11) and (A.13) are fulfilled, then

$$
\|\varphi(\widetilde{H}) J-J \varphi(H)\|_{m \rightarrow 0} \leq \eta_{\varphi}(\delta)
$$


for all $\varphi \in \mathrm{C}\left(\overline{\mathbb{R}}_{+}\right)$where $\eta_{\varphi}(\delta) \rightarrow 0$ as $\delta \rightarrow 0$.

Proof. Let $p(\lambda):=\sum_{j=0}^{n} a_{j}(\lambda+1)^{-j}$ be a polynomial in $(\lambda+1)^{-1}$. Then

$$
\begin{aligned}
& \|\varphi(\widetilde{H}) J-J \varphi(H)\|_{m \rightarrow 0} \\
& \leq\|(\varphi-p)(\widetilde{H})\|_{0 \rightarrow 0}\|J\|_{m \rightarrow 0}+\|J\|_{0 \rightarrow 0}\|(\varphi-p)(H)\|_{m \rightarrow 0} \\
& \quad+\sum_{j=0}^{n}\left|a_{j}\right|\left\|\widetilde{R}^{j} J-J R^{j}\right\|_{m \rightarrow 0} \leq 4\|\varphi-p\|_{\infty}+\sum_{j=0}^{n}\left|a_{j}\right| 4 j \delta=: \eta_{\varphi}(\delta, p)
\end{aligned}
$$

using (A.7), the spectral calculus, (A.13) and (A.17). Here, $\|\varphi\|_{\infty}$ denotes the supremum norm of $\varphi$.

Suppose $\eta>0$. By the Stone-Weierstrass theorem there exists a polynomial $p$ such that $\|p-\varphi\|_{\infty} \leq \eta / 8$. If

$$
0<\delta \leq \frac{\eta}{8 \sum_{j=0}^{n}\left|a_{j}\right| j}
$$

then $\eta_{\varphi}(\delta):=\eta_{\varphi}(\delta, p) \leq \eta / 2+\eta / 2=\eta$ and therefore $\eta_{\varphi}(\delta) \rightarrow 0$ as $\delta \rightarrow 0$.

In a second step we extend the previous result to certain bounded measurable functions $\psi: \overline{\mathbb{R}}_{+} \longrightarrow \mathbb{C}$.

Theorem A.8. Suppose that $U \subset \overline{\mathbb{R}}_{+}$and that $\psi: \overline{\mathbb{R}}_{+} \longrightarrow \mathbb{C}$ is a measurable, bounded function, continuous on $U$ such that $\lim _{\lambda \rightarrow \infty} \psi(\lambda)$ exist. Then

$$
\|\psi(\widetilde{H}) J-J \psi(H)\|_{m \rightarrow 0} \leq \eta_{\psi}(\delta)
$$

for all pairs of non-negative operators and Hilbert spaces $(H, \mathcal{H})$ and $(\widetilde{H}, \widetilde{\mathcal{H}})$ which are $\delta$-close provided

$$
\sigma(H) \subset U \quad \text { or } \quad \sigma(\widetilde{H}) \subset U .
$$

Furthermore, $\eta_{\varphi}(\delta) \rightarrow 0$ as $\delta \rightarrow 0$.

Proof. Let $\chi_{1}$ be a continuous function on $\overline{\mathbb{R}}_{+}$satisfying $0 \leq \chi_{1} \leq 1, \chi_{1}=1$ on $\sigma(H) \cup\{\infty\}$ (resp. $\chi_{1}=1$ on $\sigma(\widetilde{H}) \cup\{\infty\}$ if $U$ is a neighbourhood of $\sigma(\widetilde{H})$ ) and supp $\chi_{1} \subset U$. Then $\chi_{1} \psi$ and $\chi_{2}=1-\chi_{1}$ are continuous functions on $\overline{\mathbb{R}}_{+}$and

$$
\begin{aligned}
\| \psi(\widetilde{H}) & J-J \psi(H) \|_{m \rightarrow 0} \\
& \leq\left\|\left(\chi_{1} \psi\right)(\widetilde{H}) J-J\left(\chi_{1} \psi\right)(H)\right\|_{m \rightarrow 0}+\left\|\left(\chi_{2} \psi\right)(\widetilde{H}) J-J\left(\chi_{2} \psi\right)(H)\right\|_{m \rightarrow 0} .
\end{aligned}
$$

In the case that $U$ is a neighbourhood of $\sigma(H)$ we can estimate the norm with $\chi_{2}$ by

$$
\|\psi\|_{\infty}\left\|\chi_{2}(\widetilde{H}) J-J \chi_{2}(H)\right\|_{m \rightarrow 0}
$$

using the fact that $\left(\chi_{2} \psi\right)(H)=\chi_{2}(H)=0$ since $\chi_{2}=0$ on $\sigma(H)$.

In the case that $U$ is a neighbourhood of $\sigma(\widetilde{H})$ and if $m \geq 1$ then we can estimate the norm with $\chi_{2}$ by

$$
\left\|J\left(\chi_{2} \psi\right)(H)\right\|_{m \rightarrow 0} \leq\|J\|_{0 \rightarrow 0}\left\|\left(\chi_{2} \psi\right)(H)\right\|_{m \rightarrow 0} \leq 2\|\psi\|_{\infty}\left\|\chi_{2}(H)\right\|_{m \rightarrow 0}
$$

again using the fact that $\left(\chi_{2} \psi\right)(\widetilde{H})=\chi_{2}(\widetilde{H})=0$ since $\chi_{2}=0$ on $\sigma(\widetilde{H})$. Now

$$
\left\|\chi_{2}(H)\right\|_{m \rightarrow 0} \leq\left\|\mathbb{1}-J^{\prime} J\right\|_{1 \rightarrow 0}\left\|\chi_{2}(H)\right\|_{m \rightarrow 1}+\left\|J^{\prime}\right\|_{0 \rightarrow 0}\left\|J \chi_{2}(H)-\chi_{2}(\widetilde{H}) J\right\|_{m \rightarrow 0} \text {. }
$$


Note that $\left\|\chi_{2}(H)\right\|_{m \rightarrow 1} \leq 1$ since $m \geq 1$. If $m=0$ then use the fact that

$$
\|\psi(\widetilde{H}) J-J \psi(H)\|_{0 \rightarrow 0}=\left\|\psi(H) J^{*}-J^{*} \psi(\widetilde{H})\right\|_{0 \rightarrow 0}
$$

and argue as in the case where $\sigma(H) \subset U$ with the roles of $H$ and $\widetilde{H}$ interchanged.

Applying the preceding theorem twice (in each of the above cases), we have the error estimate

$$
\eta_{\psi}(\delta):=\eta_{\chi_{1} \psi}(\delta)+2\|\psi\|_{\infty}\left(2 \eta_{\chi_{2}}(\delta)+\delta\right)
$$

Example A.9. Consider $\psi=\mathbb{1}_{I}$ with an interval $I$ such that $\partial I \cap \sigma(H)=\emptyset$ or $\partial I \cap \sigma(\widetilde{H})=\emptyset$ then the spectral projections satisfy

$$
\left\|\mathbb{1}_{I}(\tilde{H}) J-J \mathbb{1}_{I}(H)\right\|_{m \rightarrow 0} \leq \eta_{\mathbb{1}_{I}}(\delta) .
$$

Finally we show the following estimates from the ones already considered:

Theorem A.10. Suppose that (A.10), (A.12), (A.13) and

$$
\|\varphi(\widetilde{H}) J-J \varphi(H)\|_{m \rightarrow 0} \leq \eta
$$

for some function $\varphi$ and some constant $\eta>0$. Then we have

$$
\begin{gathered}
\left\|\varphi(H) J^{\prime}-J^{\prime} \varphi(\widetilde{H})\right\|_{0 \rightarrow-m} \leq 2\|\varphi\|_{\infty} \delta+\eta \\
\left\|\varphi(H)-J^{\prime} \varphi(\widetilde{H}) J\right\|_{m \rightarrow 0} \leq C \delta+2 \eta \\
\left\|\varphi(\widetilde{H})-J \varphi(H) J^{\prime}\right\|_{0 \rightarrow 0} \leq 5 C \delta+2 \eta
\end{gathered}
$$

provided $m=0$ for the last estimate. Here, $C:=\|\varphi\|_{\infty}$ if $m \geq 1$ and $C>0$ is a constant satisfying $|\varphi(\lambda)| \leq C(\lambda+1)^{-1 / 2}$ for all $\lambda$ if $m=0$.

Proof. The first estimate follows from

$$
\left\|\varphi(H) J^{\prime}-J^{\prime} \varphi(\widetilde{H})\right\|_{0 \rightarrow-m} \leq 2\|\varphi\|_{\infty}\left\|J^{\prime}-J^{*}\right\|_{0 \rightarrow 0}+\left\|\varphi(H) J^{*}-J^{*} \varphi(\widetilde{H})\right\|_{0 \rightarrow-m}
$$

and (A.6); the second from

$$
\begin{aligned}
& \left\|\varphi(H)-J^{\prime} \varphi(\widetilde{H}) J\right\|_{m \rightarrow 0} \\
& \quad \leq\left\|\mathbb{1}-J^{\prime} J\right\|_{1 \rightarrow 0}\|\varphi(H)\|_{m \rightarrow 1}+\left\|J^{\prime}\right\|_{0 \rightarrow 0}\|J \varphi(H)-\varphi(\widetilde{H}) J\|_{m \rightarrow 0}
\end{aligned}
$$

and the third from

$$
\begin{aligned}
\| \varphi(\widetilde{H})-J \varphi(H) & J^{\prime} \|_{0 \rightarrow 0} \\
& \leq\left\|\mathbb{1}-J J^{\prime}\right\|_{1 \rightarrow 0}\|\varphi(\widetilde{H})\|_{0 \rightarrow 1}+\|J\|_{0 \rightarrow 0}\left\|J^{\prime} \varphi(\widetilde{H})-\varphi(H) J^{\prime}\right\|_{0 \rightarrow 0}
\end{aligned}
$$

together with (A.23). 
A.5. Spectral convergence. We now prove some convergence results for spectral projections and (parts) of the spectrum.

Theorem A.11. Let $I$ be a measurable and bounded subset of $\mathbb{R}$. Then there exists $\delta_{0}=\delta_{0}(I, k)>0$ such that for all $\delta>0$ we have

$$
\operatorname{dim} P=\operatorname{dim} \widetilde{P}
$$

for all pairs of non-negative operators and Hilbert spaces $(H, \mathcal{H})$ and $(\widetilde{H}, \widetilde{\mathcal{H}})$ which are $\delta$-close of order $k$ provided

$$
\partial I \cap \sigma(H)=\emptyset \quad \text { or } \quad \partial I \cap \sigma(\widetilde{H})=\emptyset .
$$

Here, $P:=\mathbb{1}_{I}(H)$ and $\operatorname{dim} P:=\operatorname{dim} P(\mathcal{H})$, similarly for $\widetilde{H}$.

Proof. Let us first show the inequality $\operatorname{dim} P \leq \operatorname{dim} \widetilde{P}$ : Suppose $f \in P(\mathcal{H})$. Then $\|f\|_{m} \leq C_{I, m}\|f\|_{0}$ with

$$
C_{I, m}:=\sup _{\lambda \in I}(1+\lambda)^{m / 2}<\infty
$$

since $I$ is bounded. Furthermore,

$$
\begin{aligned}
\|\widetilde{P} J f\|_{0} \geq\|J P f\|_{0}-\|(\widetilde{P} J-J P) f\|_{0} \\
\quad \geq\|J f\|_{0}-\|\widetilde{P} J-J P\|_{m \rightarrow 0}\|f\|_{0} \geq\left(1-\delta^{\prime} C_{I, 1}-\eta_{\mathbb{1}_{I}}(\delta)\right)\|f\|_{0}
\end{aligned}
$$

using Lemma A.4 and Theorem A.8. Since $\delta^{\prime} \rightarrow 0$ and $\eta_{\mathbb{1}_{I}}(\delta) \rightarrow 0$ as $\delta \rightarrow 0$ there exists $\delta_{0}>0$ such that

$$
\|\widetilde{P} J f\|_{0} \geq \frac{1}{2}\|f\|_{0}
$$

provided $0<\delta \leq \delta_{0}$. Therefore, $\widetilde{P} J \uparrow_{P(\mathcal{H})}$ is injective. If $f_{1}, \ldots, f_{d}$ are linear independent in $P(\mathcal{H})$, the same is true for $\widetilde{P} J f_{1}, \ldots, \widetilde{P} J f_{d}$ in $\widetilde{P}(\widetilde{\mathcal{H}})$. If $P(\mathcal{H})$ is infinite dimensional so is $\widetilde{P}(\widetilde{\mathcal{H}})$. Thus we have shown $\operatorname{dim} P \leq \operatorname{dim} \widetilde{P}$.

The other inequality is more difficult due to the asymmetry in the norm convergence $\|\cdot\|_{m \rightarrow 0}$ if $m>0$. Suppose that $u \in \widetilde{P}(\widetilde{\mathcal{H}})$ and that $\chi_{i} \in \mathrm{C}\left(\overline{\mathbb{R}}_{+}\right)$with $\chi_{1}+\chi_{2}+\chi_{3}=1$. Suppose in addition that $\operatorname{supp} \chi_{1}$ and $\operatorname{supp} \chi_{2}$ are compact, that $\operatorname{supp} \chi_{1}$ and supp $\chi_{3}$ are disjoint and that supp $\chi_{2} \cap I=\emptyset$. Then

$$
\begin{aligned}
& \left\|P J^{*} u\right\|_{-m} \geq\left\|J^{*} \widetilde{P} u\right\|_{-m}-\left\|\left(\widetilde{P} J^{*}-J^{*} \widetilde{P}\right) u\right\|_{-m} \\
& \geq\left\|\chi_{1}(H) J^{*} u\right\|_{-m}-\left\|\chi_{2}(H) J^{*} u\right\|_{-m}-\left\|\chi_{3}(H) J^{*} u\right\|_{-m}-\|\widetilde{P} J-J P\|_{m \rightarrow 0}\|u\|_{0} \\
& \quad \geq C_{I, m}^{\prime}\left\|J^{*} \widetilde{P} u\right\|_{0}-\left\|\left(\chi_{2}(H) J^{*}-J^{*} \chi_{2}(\widetilde{H})\right) \widetilde{P} u\right\|_{-m}-\eta_{\mathbb{1}_{I}}(\delta)\|u\|_{0}
\end{aligned}
$$

by Theorem A.8 and the fact that $\chi_{2}(\widetilde{H}) \widetilde{P}=\left(\chi_{2} \mathbb{1}_{I}\right)(\widetilde{H})=0$ since the support of $\chi_{2}$ and $I$ are disjoint. Here,

$$
C_{I, m}^{\prime}:=\inf _{\left\{\lambda \mid \chi_{1}(\lambda)=1\right\}}(1+\lambda)^{-m / 2}-\sup _{\lambda \in \operatorname{supp} \chi_{3}}(1+\lambda)^{m / 2}
$$

by the spectral calculus. Since $\chi_{1}$ and $\chi_{3}$ have disjoint support, $C_{I, m}^{\prime}>0$. Next, the norm involving $\chi_{2}$ can be estimated from above by

$$
\eta_{\chi_{2}}(\delta)\|u\|_{0}
$$


using Theorem A.7. Furthermore,

$$
\left\|J^{*} u\right\|_{0} \geq\left\|J^{\prime} u\right\|_{0}-\left\|\left(J^{*}-J^{\prime}\right) u\right\|_{0} \geq\left(1-C_{I, 1} \delta^{\prime}-\delta\right)\|u\|_{0}
$$

by Lemma A.4 and (A.10). Finally, we have shown that

$$
\left\|P J^{*} u\right\|_{-m} \geq\left(C_{I, m}^{\prime}\left(1-C_{I, 1} \delta^{\prime}-\delta\right)-\eta_{\chi_{2}}(\delta)-\eta_{\mathbb{1}_{I}}(\delta)\right)\|u\|_{0} .
$$

The inequality $\operatorname{dim} P \geq \operatorname{dim} \widetilde{P}$ follows as before.

In the case of 1-dimensional projections we can even show the convergence of the corresponding eigenvectors. Note that generically, the eigenvalues are simple (cf. [U76]):

Theorem A.12. Suppose that $\varphi$ is a normalised eigenvector of $H$ with eigenvalue $\lambda$ and that $\operatorname{dim} \mathbb{1}_{I}(H)=1$ for some open, bounded interval $I \subset[0, \infty)$ containing $\lambda$. Then there exists $\delta_{0}=\delta(I, k)>0$ such that $\widetilde{H}$ has only one eigenvalue $\tilde{\lambda}$ of multiplicity 1 in I for all $(\widetilde{H}, \widetilde{\mathcal{H}})$ being $\delta$-close of order $k$ to $(H, \mathcal{H})$ and all $0<\delta<\delta_{0}$.

In addition, there exist a unique eigenvector $\widetilde{\varphi}$ (up to a unitary scalar factor close to 1) and functions $\eta_{1,2}(\delta) \rightarrow 0$ as $\delta \rightarrow 0$ depending only on $\lambda$ and $k$ such that

$$
\|J \varphi-\widetilde{\varphi}\| \leq \eta_{1}(\delta), \quad\left\|J^{\prime} \widetilde{\varphi}-\varphi\right\| \leq \eta_{2}(\delta) .
$$

Proof. Denote the corresponding eigenprojections by $P$ resp. $\widetilde{P}$. The first assertion follows from Theorem A.11. For the second, note that

$$
\widetilde{\varphi}=\frac{1}{\langle\widetilde{P} J \varphi, J \varphi\rangle} \widetilde{P} J \varphi
$$

since $\widetilde{P}$ is a 1-dimensional projection. Note in addition that

$$
\langle\widetilde{P} J \varphi, J \varphi\rangle=\|\widetilde{P} J \varphi\|^{2} \geq \frac{1}{4}\|\varphi\|^{2}=\frac{1}{4}, \quad 0<\delta<\delta_{0}
$$

for some $\delta_{0}=\delta_{0}(I, k)$ due to (A.26). Now,

$$
\begin{aligned}
& \|J \varphi-\widetilde{\varphi}\|=\left\|J P \varphi-\frac{1}{\langle\widetilde{P} J \varphi, J \varphi\rangle} \widetilde{P} J \varphi\right\| \\
& \quad \leq\|(J P-\widetilde{P} J) \varphi\|+\left|1-\frac{1}{\langle\widetilde{P} J \varphi, J \varphi\rangle}\right|\|\widetilde{P} J \varphi\| \\
& \leq \eta_{\mathbb{1}_{I}}(\delta)+8\left|\langle(\widetilde{P} J-J P) \varphi, J \varphi\rangle+\|J \varphi\|^{2}-\|\varphi\|^{2}\right| \leq 17 \eta_{\mathbb{1}_{I}}(\delta)+3 \delta=: \eta_{1}(\delta)
\end{aligned}
$$

since $\varphi=P \varphi$ and $\|\varphi\|=1$ using (A.13) and (A.14). The second estimate follows immediately from

$$
\left\|J^{\prime} \widetilde{\varphi}-\varphi\right\| \leq\left\|J^{\prime}(\widetilde{\varphi}-J \varphi)\right\|+\left\|\left(J^{\prime} J-\mathbb{1}\right) \varphi\right\| \leq 2 \eta_{1}(\delta)+\delta(1+\lambda)=: \eta_{2}(\delta) .
$$

All estimates are valid for $0<\delta<\delta_{0}$. Note that $\delta_{0}$ and $\eta_{i}(\delta)$ depend also on $I$ and therefore on $\lambda$.

We now show that the spectrum of the resolvents $R=(H+1)^{-1}$ and $\widetilde{R}=$ $(\widetilde{H}+1)^{-1}$ are close in the Hausdorff distance defined by

$$
d(A, B):=\max \left\{\sup _{a \in A} d(a, B), \sup _{b \in B} d(b, A)\right\}
$$


for subsets $A, B$ of $\mathbb{R}$ where $d(a, B):=\inf _{b \in B}|a-b|$. Furthermore, we set ${ }^{4}$

$$
\bar{d}(A, B):=d\left((A+1)^{-1},(B+1)^{-1}\right)
$$

for closed subsets of $[0, \infty)$ (cf. also [HeN99, Appendix A], where an equivalent characterisation of the convergence $\bar{d}\left(A_{n}, A\right) \rightarrow 0$ as $n \rightarrow \infty$ is given).

Theorem A.13. There exists $\eta(\delta)>0$ with $\eta(\delta) \rightarrow 0$ as $\delta \rightarrow 0$ such that

$$
\bar{d}\left(\sigma_{\bullet}(H), \sigma_{\bullet}(\widetilde{H})\right) \leq \eta(\delta)
$$

for all pairs of non-negative operators and Hilbert spaces $(H, \mathcal{H})$ and $(\widetilde{H}, \widetilde{\mathcal{H}})$ which are $\delta$-close. Here, $\sigma_{\bullet}(H)$ denotes either the entire spectrum, the essential or the discrete spectrum of $H$.

Furthermore, the multiplicity of the discrete spectrum is preserved, i.e., if $\lambda \in$ $\sigma_{\text {disc }}(H)$ has multiplicity $m>0$ then $\operatorname{dim} \mathbb{1}_{I}(\widetilde{\mathcal{H}})=m$ for $I:=(\lambda-\eta(\delta), \lambda+\eta(\delta))$ provided $\delta$ is small enough.

Proof. We start with the discrete spectrum. Let $\eta>0$ and $z=(\lambda+1)^{-1}>0$, $\lambda \in \sigma_{\text {disc }}(H)$. By the definition of the discrete spectrum, there exists an open interval $I$ containing $\lambda$ such that $I \cap \sigma(H)=\{\lambda\}$ and $0<\operatorname{dim} \mathbb{1}_{I}(H)<\infty$. Without loss of generality, we assume that $I \subset(\lambda-\eta, \lambda+\eta)$. From Theorem A.11 it follows that $\operatorname{dim} \mathbb{1}_{I}(H)=\operatorname{dim} \mathbb{1}_{I}(\widetilde{H})$ provided $0<\delta<\delta_{z}$ for some $\delta_{z}>0$. In particular, the multiplicity is preserved and there exists $\widetilde{\lambda} \in I \cap \sigma_{\text {disc }}(\widetilde{H})$, i.e.,

$$
d(z, \widetilde{S}) \leq|z-\widetilde{z}| \leq|\lambda-\widetilde{\lambda}|<\eta
$$

where $\widetilde{S}=\left(\sigma_{\text {disc }}(\widetilde{H})+1\right)^{-1}$ and $\widetilde{z}=(\widetilde{\lambda}+1)^{-1}$. Now let $\delta(\eta)$ be the minimum of all $\delta_{z}$ where $z$ runs through the finite set $S \cap[\eta, 1]$ with $S:=\left(\sigma_{\text {disc }}(H)+1\right)^{-1}$. Then A.29 holds for all $z \in S \cap[\eta, 1]$ and $0<\delta<\delta(\eta)$. If $\sigma_{\text {disc }}(H)$ is finite, we just have to assure that $\eta<\inf S$. If $\sigma_{\text {disc }}(H)$ is infinite, so is $\sigma_{\text {disc }}(\widetilde{H})$ and in particular, $\widetilde{S} \cap(0, \eta) \neq \emptyset$ for all $\eta>0$. Therefore, if $z \in(0, \eta) \cap S$ then $d(z, \widetilde{S}) \leq \eta$. Finally, (A.29) holds for all $z \in S$ and $0<\delta<\delta(\eta)$.

Interchanging the roles of $H$ and $\widetilde{H}$ leads to the inequality $d(\widetilde{z}, S) \leq \eta$ for all $\widetilde{z} \in \widetilde{S}$ and therefore $\bar{d}\left(\sigma_{\text {disc }}(H), \sigma_{\text {disc }}(\widetilde{H})\right) \leq \eta(\delta)$ where $\eta(\delta)$ is the smallest constant satisfying the previous estimate for all $(H, \mathcal{H}),(\widetilde{H}, \widetilde{\mathcal{H}})$ being $\delta$-close.

For the essential spectrum we argue similarly: Let $\eta>0$ and $z=(\lambda+1)^{-1}>0$ with $\lambda \in \sigma_{\text {ess }}(H)$. Let $I$ be an open interval with $\lambda \in I$ and $\partial I \cap \sigma(H)=\emptyset$. If $I$ can be chosen in such a way that $I \subset(\lambda-\eta, \lambda+\eta)$ then $\infty=\operatorname{dim} \mathbb{1}_{I}(H)=\operatorname{dim} \mathbb{1}_{I}(\widetilde{H})$ for all pairs $(\widetilde{H}, \widetilde{\mathcal{H}})$ being $\delta$-close, $0<\delta<\delta_{z}$ for some fixed $\delta_{z}>0$ due to Theorem A.11. In particular, $I \cap \sigma_{\text {ess }}(\widetilde{H}) \neq \emptyset$ and therefore $d(z, \widetilde{S})<\eta$ as in (A.29) where now $\widetilde{S}=\left(\sigma_{\text {ess }}(\widetilde{H})+1\right)^{-1}$.

If no such interval $I$ exist, then there is $0<\eta_{0}<\eta$ such that $I_{0}:=\left(\lambda-\eta_{0}, \lambda+\right.$ $\left.\eta_{0}\right) \subset \sigma_{\text {ess }}(H)$. We want to show that in this case, $I_{0} \subset \sigma_{\text {ess }}(\widetilde{H})$ and in particular, $d(z, \widetilde{S}) \leq \eta_{0}<\eta$ provided $0<\delta<\delta_{z}$ for some fixed $\delta_{z}$ : Suppose that this is not true. Then there were $\widetilde{\lambda} \in I_{0}$ and an open interval $J$ containing $\widetilde{\lambda}$ which is disjoint from the closed set $\sigma_{\text {ess }}(\widetilde{H})$ for all $\delta>0$ and all $(\widetilde{H}, \widetilde{\mathcal{H}})$ being $\delta$-close.

\footnotetext{
${ }^{4}$ Strictly speaking, $(\sigma(H)+1)^{-1}=\sigma(R) \backslash\{0\}$, but the point 0 plays no special role since $d(A, B)=d(\bar{A}, \bar{B})$.
} 
But then, Theorem A.11 implies $0=\operatorname{dim} \mathbb{1}_{J}(\widetilde{H})=\operatorname{dim} \mathbb{1}_{J}(H)$ contradicting the fact that $J \subset \sigma_{\text {ess }}(H)$.

A compactness argument shows that there exists $\delta(\eta)>0$ such that (A.29) is true for all $z$ in the compact set $S \cap[\eta, 1]$ and all $(\widetilde{H}, \widetilde{\mathcal{H}})$ being $\delta$-close, $\delta<\delta(\eta)$ where $S=\left(\sigma_{\text {ess }}(H)+1\right)^{-1}$. If $\sigma_{\text {ess }}(H)$ is bounded (from above) then $S \cap[\eta, 1]=S$ provided $\eta<\inf S$ and we are done. If $\sigma_{\text {ess }}(H)$ is unbounded, a similar reasoning as before shows that the same is true for $\sigma_{\text {ess }}(\widetilde{H})$. In particular, $(0, \eta) \cap \widetilde{S} \neq \emptyset$ and $d(z, \widetilde{S})<\eta$ for $z \in(0, \eta) \cap S$, i.e., A.29) holds for all $z \in S$. The assertion follows as in the discrete case by symmetry.

The case of the entire spectrum can be shown similarly.

We have the following immediate consequences when $\sigma_{\text {disc }}(H)=\emptyset$ resp. $\sigma_{\text {ess }}(H)=\emptyset$ :

Corollary A.14. Suppose that $H$ has purely essential spectrum. Then for each $\lambda \in \sigma_{\text {ess }}(H)$ there is essential spectrum close to $\lambda$ for $\widetilde{H}$ being $\delta$-close to $H$. Either $\widetilde{H}$ has no discrete spectrum or the discrete spectrum merges into the essential spectrum as $\delta \rightarrow 0$.

Corollary A.15. Suppose that $H$ has purely discrete spectrum denoted by $\lambda_{k}$ (repeated according to multiplicity). Then the infimum of the essential spectrum of $\widetilde{H}$ tends to infinity (if there where any) and there exists $\eta_{k}(\delta)>0$ with $\eta_{k}(\delta) \rightarrow 0$ as $\delta \rightarrow 0$ such that

$$
\left|\lambda_{k}-\widetilde{\lambda}_{k}\right| \leq \eta_{k}(\delta)
$$

for all $(\widetilde{H}, \widetilde{\mathcal{H}})$ being $\delta$-close. Here, $\widetilde{\lambda}_{k}$ denotes the discrete spectrum of $\widetilde{H}$ (below the essential spectrum) repeated according to multiplicity.

Note that the convergence $\eta_{k}(\delta) \rightarrow 0$ is not uniform in $k$. The convergence of the eigenvalues can also be seen by a direct argument using the min-max principle:

Remark A.16. If we assume that

$$
\begin{aligned}
\mathfrak{h}(f) & \geq \widetilde{\mathfrak{h}}\left(J_{1} f\right)-\delta\|f\|_{1}^{2}, & & \widetilde{\mathfrak{h}}(u) \geq \mathfrak{h}\left(J_{1}^{\prime} u\right)-\delta\|u\|_{1}^{2}, \\
\|f\|^{2} & \geq\left\|J_{1} f\right\|^{2}+\delta\|f\|_{1}^{2}, & & \|u\|^{2} \geq\left\|J_{1}^{\prime} u\right\|^{2}+\delta\|u\|_{1}^{2}
\end{aligned}
$$

we obtain the more concrete eigenvalue estimate

$$
\left|\lambda_{k}-\widetilde{\lambda}_{k}\right| \leq \frac{\left(\lambda_{k}+2+\frac{\left(\lambda_{k}+2\right)^{2}}{1-\delta\left(\lambda_{k}+1\right)} \delta\right)^{2}}{1-\delta\left(\lambda_{k}+1+\frac{\left(\lambda_{k}+2\right)^{2}}{1-\delta\left(\lambda_{k}+1\right)} \delta\right)} \cdot \delta=O(\delta)
$$

using the min-max principle where $O(\delta)$ depends on $\lambda_{k}$ (cf. [EP05, Lemma 2.1]). Note that the assumptions (A.31) and (A.32) are equivalent to the estimates

$$
\begin{aligned}
H-J_{1}^{*} \widetilde{H} J_{1}+\delta(H+1) & \geq 0, & \widetilde{H}-J_{1}^{*} H J_{1}^{\prime}+\delta(\widetilde{H}+1) & \geq 0, \\
J_{1}^{*} J_{1}-\mathbb{1}+\delta(H+1) & \geq 0, & J_{1}^{\prime *} J_{1}^{\prime}-\mathbb{1}+\delta(\widetilde{H}+1) & \geq 0
\end{aligned}
$$

in the sense that $A: \mathcal{H}_{1} \longrightarrow \mathcal{H}_{-1} \geq 0$ iff $\langle A f, f\rangle \geq 0$ for all $f \in \mathcal{H}_{1}$ and similarly on $\widetilde{\mathcal{H}}$. Note that A.31 and (A.32) do not follow from the closeness assumptions (A.9) - A.13); e.g. for (A.32) one needs in addition that $\left\|J_{1}\right\|_{1 \rightarrow 1} \leq C$ for some constant $C>0$ and similarly for $J_{1}^{\prime}$. The estimates (A.31)-(A.32) have 
been used e.g. in EP05, KuZ01, RS01 in the graph model and the verification of (A.31) - A.32) is quite similar to the proof of the closeness assumptions (A.9)(A.13) as we have seen in Section 2.

Acknowledgments. It is a pleasure to thank Pavel Exner, Peter Hislop, Fernando Lledó, Gianfausto Dell'Antonio and Luca Tenuta for fruitful discussions and helpful comments on this manuscript. Part of this work has been done while the author was funded by the DFG under the grant Po-1034/1-1.

\section{REFERENCES}

[AADH94] S. Alama, M. Avellaneda, P.A. Deift, and R. Hempel, On the existence of eigenvalues of a divergence-form operator $A+\lambda B$ in a gap of $\sigma(A)$, Asymptotic Anal. 8 (1994), 311-344.

[AEL94] J. E. Avron, P. Exner, and Y. Last, Periodic Schroedinger operators with large gaps and Wannier-Stark ladders, Phys. Rev. Lett. 72 (1994), no. 6, 896-899.

[AGHH05] S. Albeverio, F. Gesztesy, R. Høegh-Krohn, and H. Holden, Solvable models in quantum mechanics, second ed., AMS Chelsea Publishing, Providence, RI, 2005.

[AS00] M. Aizenman and J. H. Schenker, The creation of spectral gaps by graph decoration, Lett. Math. Phys. 53 (2000), 253-262.

[BCG01] Martin Barlow, Thierry Coulhon, and Alexander Grigor'yan, Manifolds and graphs with slow heat kernel decay, Invent. Math. 144 (2001), no. 3, 609-649.

[BEG03] J. Brüning, P. Exner, and V. A. Geyler, Large gaps in point-coupled periodic systems of manifolds, J. Phys. A 36 (2003), no. 17, 4875-4890. MR MR1984016

[BGL05] J. Brüning, V. Geyler, and I. Lobanov, Spectral properties of Schrödinger operators on decorated graphs, Mat. Zametki 77 (2005), no. 1, 152-156.

[Br81a] R. Brooks, The fundamental group and the spectrum of the Laplacian, Comment. Math. Helv. 56 (1981), 581-598.

[Br81b] _ A relation between growth and the spectrum of the Laplacian, Math. $\mathrm{Z}$. 178 (1981), no. 4, 501-508.

[Ca97] Carla Cattaneo, The spectrum of the continuous Laplacian on a graph, Monatsh. Math. 124 (1997), no. 3, 215-235.

[DaS92] E. B. Davies and B. Simon, Spectral properties of Neumann Laplacian of horns, Geom. Funct. Anal. 2 (1992), no. 1, 105-117.

[EvHa89] W. D. Evans and D. J. Harris, On the approximation numbers of Sobolev embeddings for irregular domains, Quart. J. Math. Oxford Ser. (2) 40 (1989), no. 157, $13-42$.

[EP05] P. Exner and O. Post, Convergence of spectra of graph-like thin manifolds, Journal of Geometry and Physics 54 (2005), 77-115.

[EP06]__ Convergence of resonances on thin branched quantum wave guides, (in preparation) (2006).

[EvS00] W. D. Evans and Y. Saitō, Neumann Laplacians on domains and operators on associated trees, Q. J. Math. 51 (2000), no. 3, 313-342.

[E95] P. Exner, Lattice Kronig-Penney models, Phys. Rev. Lett. 74 (1995), no. 18, 3503-3506.

[HeN99] I. Herbst and S. Nakamura, Schrödinger operators with strong magnetic fields: Quasi-periodicity of spectral orbits and topology, American Mathematical Society. Transl., Ser. 2, Am. Math. Soc. 189(41) (1999), 105-123.

[KaP88] L. Karp and M. Pinsky, First-order asymptotics of the principal eigenvalue of tubular neighborhoods, Geometry of random motion (Ithaca, N.Y., 1987), Contemp. Math., vol. 73, Amer. Math. Soc., Providence, RI, 1988, pp. 105-119.

[KoS99] V. Kostrykin and R. Schrader, Kirchhoff's rule for quantum wires, J. Phys. A 32 (1999), no. 4, 595-630.

[Ku02] P. Kuchment, Graph models for waves in thin structures, Waves Random Media 12 (2002), no. 4, R1-R24. 
[Ku04] _ Quantum graphs: I. Some basic structures, Waves Random Media 14 (2004), S107-S128.

[Ku05] Q Quantum graphs. II. Some spectral properties of quantum and combinatorial graphs, J. Phys. A 38 (2005), no. 22, 4887-4900.

[KuZ01] P. Kuchment and H. Zeng, Convergence of spectra of mesoscopic systems collapsing onto a graph, J. Math. Anal. Appl. 258 (2001), no. 2, 671-700.

[KuZ03] - Asymptotics of spectra of Neumann Laplacians in thin domains, Advances in differential equations and mathematical physics (Birmingham, AL, 2002), Contemp. Math., vol. 327, Amer. Math. Soc., Providence, RI, 2003, pp. 199-213.

[Lo01] J. Lott, On the spectrum of a finite-volume negatively-curved manifold, Amer. J. Math. 123 (2001), no. 2, 185-205.

[LP04] F. Lledó and O. Post, Generating spectral gaps by geometry, arXiv:math-ph/0406032 (2004).

[LP05] Existence of spectral gaps, covering manifolds and residually finite groups, arXiv:math-ph/0503005 (2005).

[M68] J. Milnor, A note on curvature and fundamental group, J. Differential Geometry 2 (1968), 1-7.

[P03a] O. Post, Eigenvalues in spectral gaps of a perturbed periodic manifold, Mathematische Nachrichten 261-262 (2003), 141-162.

[P03b] _ Periodic manifolds with spectral gaps, J. Diff. Equations 187 (2003), 2345.

[P05] - Branched quantum wave guides with Dirichlet boundary conditions: the decoupling case, Journal of Physics A: Mathematical and General 38 (2005), no. $22,4917-4931$.

[RuS53] K. Ruedenberg and C. W. Scherr, Free-electron network model for conjugated systems, I. Theory, J. Chem. Phys. 21 (1953), 1565-1581.

[RS80] M. Reed and B. Simon, Methods of modern mathematical physics I: Functional analysis, Academic Press, New York, 1980.

[RS01] J. Rubinstein and M. Schatzman, Variational problems on multiply connected thin strips. I. Basic estimates and convergence of the Laplacian spectrum, Arch. Ration. Mech. Anal. 160 (2001), no. 4, 271-308.

[Sa00] Y Saito, The limiting equation for Neumann Laplacians on shrinking domains., Electron. J. Differ. Equ. 31 (2000), 25 p.

[T98] Alexander Teplyaev, Spectral analysis on infinite Sierpiński gaskets, J. Funct. Anal. 159 (1998), no. 2, 537-567.

[U76] K. Uhlenbeck, Generic properties of eigenfunctions, Amer. J. Math. 98 (1976), no. 4, 1059-1078.

Department of Mathematics, University of Kentucky, 751 Patterson OfFice Tower, Lexington, Kentucky 40506-0027, USA

E-mail address: post@ms.uky.edu 